\title{
Electrolysis-Driven and Pressure-Controlled Diffusive Growth of Successive Bubbles on Microstructured Surfaces
}

\author{
Peter van der Linde,,$^{\dagger}$ Álvaro Moreno Soto, ${ }^{\ddagger}$ (i) Pablo Peñas-López, ${ }^{\S}{ }^{\circledR}$ Javier Rodríguez-Rodríguez, $^{\S}$
} Detlef Lohse, ${ }^{\ddagger}$ Han Gardeniers, ${ }^{\dagger}$ Devaraj van der Meer, ${ }^{*}{ }^{\ddagger}$ and David Fernández Rivas ${ }^{\dagger}$.

${ }^{\dagger}$ Mesoscale Chemical Systems Group, MESA+ Institute and Faculty of Science and Technology and ${ }^{\ddagger}$ Physics of Fluids Group, Faculty of Science and Technology, Max Planck - University of Twente Center for Complex Fluid Dynamics, MESA+ Institute, and J. M. Burgers Centre for Fluid Dynamics, University of Twente, P.O. Box 217, 7500AE Enschede, The Netherlands

${ }^{\S}$ Fluid Mechanics Group, Universidad Carlos III de Madrid, Avda. de la Universidad 30, 28911 Leganés Madrid, Spain

\section{Supporting Information}

ABSTRACT: Control over the bubble growth rates forming on the electrodes of water-splitting cells or chemical reactors is critical with respect to the attainment of higher energy efficiencies within these devices. This study focuses on the diffusion-driven growth dynamics of a succession of $\mathrm{H}_{2}$ bubbles generated at a flat silicon electrode substrate. Controlled nucleation is achieved by means of a single nucleation site consisting of a hydrophobic micropit etched within a micrometer-sized pillar. In our experimental configuration of constant-current electrolysis, we identify gas depletion from (i) previous bubbles in the succession, (ii) unwanted bubbles forming on the sidewalls, and (iii) the mere presence of the circular cavity where the electrode is being held. The impact of these effects on bubble growth is discussed with support from numerical simulations. The time evolution of the dimensionless bubble growth coefficient, which is a measure of the overall growth rate of a particular bubble, of electrolysis-generated bubbles is compared to that of $\mathrm{CO}_{2}$ bubbles growing on a similar surface in the presence of a supersaturated solution of carbonated water. For electrolytic bubbles and under the range of current densities considered here $\left(5-15 \mathrm{~A} / \mathrm{m}^{2}\right)$, it is observed that $\mathrm{H}_{2}$ bubble successions at large gas-evolving substrates first experience a stagnation regime, followed by a fast increase in the growth coefficient before a steady state is reached. This clearly contradicts the common assumption that constant current densities must yield time-invariant growth rates. Conversely, for the case of $\mathrm{CO}_{2}$ bubbles, the growth coefficient successively decreases for every subsequent bubble as a result of the persistent depletion of dissolved $\mathrm{CO}_{2}$.

\section{INTRODUCTION}

At sufficiently large voltages, gas bubbles are produced on the electrodes in electrochemical reactors, ${ }^{1-3}$ in water-splitting cells during the electrolysis of water for hydrogen production, ${ }^{4}$ in the study of nanobubbles, ${ }^{5-7}$ and during photoelectrolysis by solar-driven cells, ${ }^{8,9}$ a topic that recently has gained interest. The formation of unwanted nucleation sites or large bubble departure sizes may lead to an excessive coverage of electrodes by bubbles. This ultimately results in increased electrical resistance ${ }^{4,10}$ within the aforementioned devices. Thus, proper control over the nucleation, growth, and detachment of bubbles is expected to play a key role in the progress toward higher energy efficiencies. ${ }^{11}$ Moreover, such control should allow us to efficiently harvest the produced gas bubbles ${ }^{12}$ without further need of energy input, e.g., in the form of pumping- or vibrationbased mechanical systems.

Control over the bubble growth rates is a critical aspect that should be achieved after properly understanding the underlying physical problem. Bubble growth kinetics in electrolytic systems has been generally described in the form of ${ }^{13-21}$

$$
R=b t^{\alpha}
$$

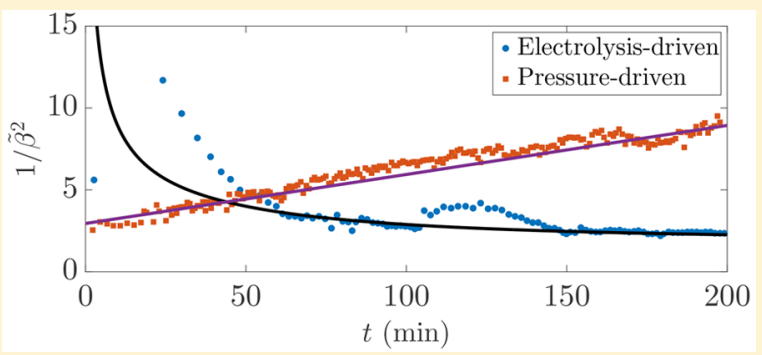

where $R$ is the bubble radius and $t$ is the actual residence time of the bubble, which starts growing at $t=0$, on the electrode before detachment. Here, $b$ is the dimensional growth coefficient whereas $\alpha$ is the scaling exponent. Throughout the majority of the bubble lifetime, except for a very fast inertiacontrolled initial growth ${ }^{15}$ (on the order of $0.1 \mathrm{~s}$ in our experiments), $b$ and $\alpha$ can be approximated as constants. The scaling exponent $\alpha$ is typically $1 / 2$ or $1 / 3$, depending on the experimental conditions.

The occurrence of one scaling exponent is determined by a quantity of immediate interest, $A_{\mathrm{e}} / R_{\mathrm{d}}{ }^{2}$, namely, the ratio of the active surface area, $A_{\mathrm{e}}$, of the electrode upon which the bubble grows to the characteristic bubble surface at the moment of detachment (at a bubble radius of $R_{\mathrm{d}}$ ). This coefficient represents the ratio between the characteristic time of the diffusive transport of the evolved gas across a region of the size of the electrode, $t_{\mathrm{t}} \approx A_{e} / D$, and that of the diffusive transport of gas to the bubble, namely, $t_{\mathrm{d}} \approx R_{\mathrm{d}}{ }^{2} / D$, which in turn limits the

Received: August 22, 2017

Revised: October 6, 2017

Published: October 17, 2017 
bubble growth rate. Here, $D$ denotes the gas diffusivity in the liquid. Alternatively, if the exsolution of dissolved gas into the bubble is considered to be a chemical reaction, then the quantity $A_{\mathrm{e}} / R_{\mathrm{d}}{ }^{2}$ can be interpreted as a Damköhler number representing the ratio between the diffusive transport time to the bulk fluid and the diffusive transport time of gas into the bubble. This ratio is geometrically imposed by the experimental configuration, and it essentially specifies the growth mechanism that determines the nature of $b$ and $\alpha .^{21}$ Two limiting cases ${ }^{14,21}$ are to be identified:

(i) Surface-reaction-controlled (or production/reactionlimited) growth for relatively small active electrode surfaces, $A_{\mathrm{e}} / R_{\mathrm{d}}{ }^{2} \ll 1$, where $R=b t^{1 / 3}$.

(ii) Diffusion-controlled (or diffusion-limited) growth for relatively large active electrode surfaces: $A_{\mathrm{e}} / R_{\mathrm{d}}{ }^{2} \gg 1$, where $R=b t^{1 / 2}$.

Bubbles generated at microelectrodes ${ }^{15,17,20}$ by catalysis and at laser-irradiated photoelectrodes ${ }^{21,22}$ or plasmonic nanoparticles $^{23}$ are common scenarios of case (i). Here, bubble growth is directly limited by the gas production rate and hence by the supplied current. Essentially, all of the gas produced at the electrode surface is directly transferred into the bubble adhered to it by diffusion and by coalescence with smaller (nano)bubbles that nucleate at the rim of the microelectrode. $^{20,23}$ Gas diffusion into the bulk electrolyte is negligible in comparison. The gas evolution efficiency, ${ }^{24}$ namely, the fraction of gas contained within the departing bubble(s) with respect to the total amount of gas produced at the electrode, has been reported to be close to $100 \% .^{20}$ For these reasons, microelectrodes enable the production of wellcontrolled successions of single bubbles that nucleate, grow, and detach in a highly periodic and repetitive fashion. In the case of a single microelectrode, multiple bubble interactions or bubble coalescence events are suppressed. Moreover, the effect of convection induced by the detachment of previous bubbles on the growth rate is negligible because viscous dissipation limits detachment-induced flow to a short time interval of around $0.1 \mathrm{~s}^{20}$ The mathematical formulation of the growth dynamics in case (i) is trivial. Provided that the growing bubble remains spherical as it grows and that the contact angle is small, a simple mass balance yields the following experimentally confirmed relation ${ }^{15,17,20,25}$

$$
R=b t^{1 / 3}=\frac{3 R_{\mathrm{u}} T_{\infty} I}{4 \pi F v P_{0}} t^{1 / 3}
$$

where $R_{\mathrm{u}}$ is the universal gas constant, $T_{\infty}$ and $P_{0}$ are the liquid pressure and temperature, $I$ is the electric current, $F$ is Faraday's constant, and $v$ is the number of electrons transferred in the electrochemical cell per molecule of evolved gas.

The mechanism behind case (ii), the case of bubbles growing on electrodes with relatively large surfaces, is substantially different. The growth is almost entirely driven by the Fickian diffusion of gas into the bubble from the surrounding supersaturated electrolyte. Mass is now transferred across the entire bubble surface. Direct gas diffusion from the substrate to the bubble is, on the other hand, usually small in comparison. We then expect very similar behavior to the diffusive, quasistatic growth of bubbles in uniformly supersaturated solutions. ${ }^{26,27}$ Diffusion-limited growth has been found to be well described by the classical theories proposed by Epstein and Plesset $^{28}$ or Scriven. ${ }^{29}$ These theories, which can be extended to the growth of surface (nano)bubbles, ${ }^{30}$ coincide in that diffusive growth may be described by $R=b t^{1 / 2}$. The scaling $R \propto t^{1 / 2}$ is in full agreement with reported electrolysis experiments for case (ii). ${ }^{13,16,18,19}$

The diffusive growth coefficient $b$ is now determined by the local degree of supersaturation of the nearby liquid, by the gas solubility, and by the gas diffusivity. The local degree of supersaturation and, as a consequence, the average bubble growth rate and gas evolution efficiency are all expected to increase with the current density. ${ }^{24}$ Most studies reporting bubble growth dynamics ${ }^{13,14,16,21}$ or analyzing mass transfer from electrodes ${ }^{24,31,32}$ assume a constant, time-independent value of the local supersaturation for a given current density. This therefore would entail a unique growth coefficient $b$ common to all bubbles, regardless of the time of nucleation after the onset of electrolysis. However, it follows that the gas supersaturation near the electrode depends not only on the current density but also on the elapsed time of electrolysis. This stands to reason because from simple mathematical considerations ${ }^{18}$ the imposition of a constant gas flux at the electrode surface must lead to an accumulation over time of dissolved gas near the electrode. The concentration of dissolved gas increases with time until an approximately steady-state profile is reached. Consequently, $b$ is expected to increase for subsequent bubbles until a steady-state value is reached, even under the imposition of a constant current density.

The constant-electrolysis scenario has some similarities to the diffusion-driven growth of a succession of bubbles from a nucleation site in a supersaturated liquid. In both scenarios, the behavior $R=b t^{1 / 2}$ holds for each individual bubble, and the value of the coefficient $b$ is observed to vary discretely from bubble to bubble in the succession. However, in the latter scenario the growth coefficient $b$ of subsequent bubbles continuously gets smaller and smaller, as opposed to becoming increasingly larger as seen during electrolysis. This effect has been recently studied for a succession of $\mathrm{CO}_{2}$ bubbles in carbonated water kept at constant pressure under supersaturation conditions. ${ }^{33}$

With all of these ideas in mind, the aim of this work is to explore in detail the time evolution of the growth coefficient for a succession of $\mathrm{H}_{2}$ bubbles produced by constant-current electrolysis. In our configuration, the electrode is much larger than the bubble, and hence $R=b t^{1 / 2}$ is expected. We make use of flat silicon electrodes with a single microstructure upon which a succession of single bubbles may form. In this way, the effect on $b$ of bubble electrode coverage, convection, and diffusive interactions between multiple bubbles ${ }^{32}$ is intentionally minimized. This is done in view of future applications in photoelectrolysis, where silicon is becoming an increasingly popular material for photoelectrodes, ${ }^{34,35}$ considering that it may be feasibly etched with micrometer- or nanometer-sized pillars ${ }^{36}$ as a means to increase the light-harvesting efficiencies and gas production rates. Finally, some interesting conclusions will be drawn by comparing the growth coefficient of electrolysis bubbles with that of $\mathrm{CO}_{2}$ bubbles in carbonated water in a pressure-controlled ( $\mathrm{P}-\mathrm{C})$ setup.

\section{EXPERIMENTS}

Microstructured Substrates. A single active site for bubble formation was defined at the center of flat boron-doped silicon substrates $\left(10 \times 10 \times 0.525 \mathrm{~mm}^{3}\right)$. This nucleation site, fabricated through reactive ion etching after photolitography, consists of a micrometer-sized hydrophobic cylindrical pit $^{37}$ (about $20 \mu \mathrm{m}$ deep) 
etched within a cylindrical micrometer-sized pillar. ${ }^{36}$ Details of the fabrication process may be found elsewhere. ${ }^{11}$

Figure 1 shows a scanning electron microscope image of a micropillar containing the superhydrophobic pit. The pit radius, $R_{0}$,

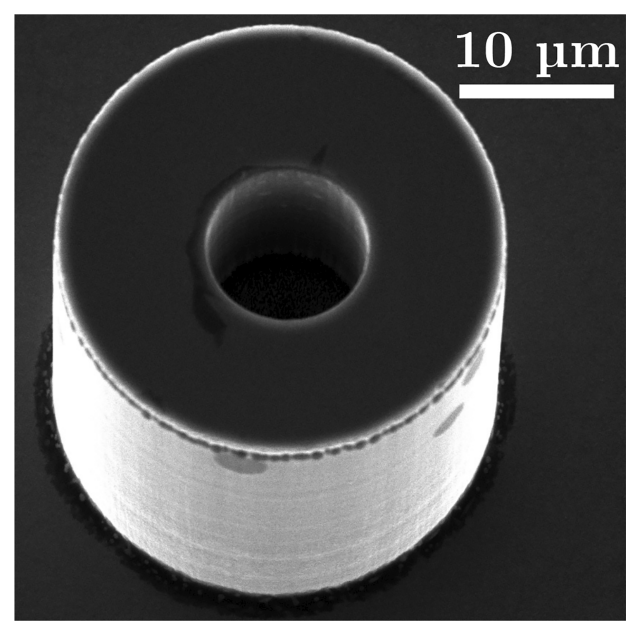

Figure 1. SEM image of a micropillar protruding from the silicon substrate. In this particular sample, the pillar height is $H_{\mathrm{p}}=30 \mu \mathrm{m}$, the pit (inner) radius is $R_{0}=5 \mu \mathrm{m}$, and the pillar (outer) radius is $R_{\mathrm{p}}=15$ $\mu \mathrm{m}$.

was set in the range of $1-10 \mu \mathrm{m}$ over the various experimental samples, values that ensure successful bubble nucleation. ${ }^{38}$ Similarly, the pillar radius, $R_{\mathrm{p}}$, was set to $5-15 \mu \mathrm{m}$. The pillar heights considered were $H_{\mathrm{p}}=0,15$, and $30 \mu \mathrm{m}$, in view of potentially identifying any influence that the distance from the electrode to the bubble nucleation spot may have on bubble growth. The case $H_{\mathrm{p}}=0 \mu \mathrm{m}$ corresponds to a micropit in an otherwise flat substrate; i.e., the pillar is absent in this case.

Experimental Method. The experimental setup, sketched in Figure 2, comprises a 3D-printed acrylic holder and a mounting plate designed to hold the silicon substrate (electrode) in place. The substrate is at the base of a circular cavity (of radius $R_{\mathrm{e}}=3.5 \mathrm{~mm}$ and depth $H_{c}=2 \mathrm{~mm}$ ) that determines the active electrode surface in contact with the electrolyte. A rubber ring, placed between the silicon

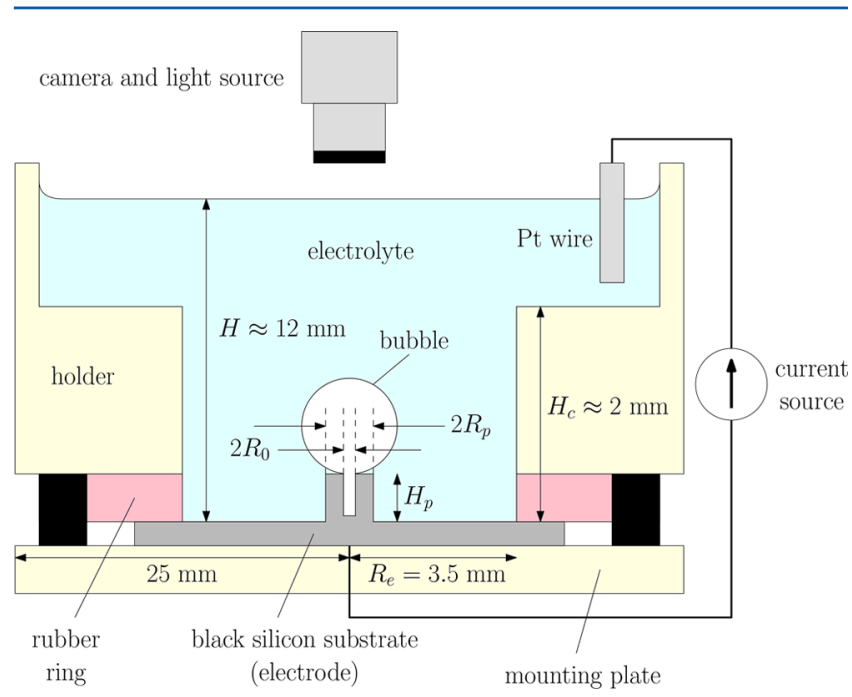

Figure 2. Sketch of the electrolysis setup. The dimensions of the substrate, cavity, and holder are not drawn to scale to highlight, within a single schematic, the different dimensions playing a crucial role in electrolytic bubble growth. Note that the pit and pillar are much smaller in reality. substrate and the substrate holder, creates a liquid-tight seal. A counter electrode in the form of a platinum wire is then placed at the corner of the holder far away from the substrate.

At the beginning of each experiment, the holder is filled with $20 \mathrm{~mL}$ of fresh electrolyte. The resulting electrolyte level depth is $H \approx 12$ $\mathrm{mm}$. The electrolyte consists of a solution composed of nondegassed Milli-Q water with $10 \mathrm{mM} \mathrm{Na}_{2} \mathrm{SO}_{4}$ salt and a $\mathrm{pH} 3$ buffer (sodium acetate anhydrous and acetic acid), giving the bubbles a neutral electric charge. ${ }^{15}$ During each experiment, a constant current $I$ in the range of 200-600 $\mu \mathrm{A}$ is supplied. The corresponding current density, computed as $j=I /\left(\pi R_{\mathrm{e}}{ }^{2}\right)$, falls in the range of $5-15 \mathrm{~A} / \mathrm{m}^{2}$. In this way, a constant molar flux of hydrogen gas, $J_{\mathrm{w}}=j /(F v)$, is uniformly released from the substrate by electrolysis. Here, $F=96485 \mathrm{C} / \mathrm{mol}$ is Faraday's constant, and $v=2$ is the number of electrons taken up per molecule of evolved $\mathrm{H}_{2}$ gas.

A few seconds after the start of electrolysis, the electrolyte is sufficiently saturated with hydrogen and a single bubble grows from the pit on top of the micropillar. Eventually, the bubble reaches a critical size and detaches. Shortly afterward, a new bubble nucleates and the process starts over again. Images of the succession of growing bubbles are acquired from the top by a digital camera mounted with an axial light source for $1-5 \mathrm{~h}$.

The radius of the gas-evolving substrate is about 10 times larger than the typical detachment radius of the bubble $\left(R_{\mathrm{d}}<0.4 \mathrm{~mm}\right)$, which gives a Damköhler number of $D_{\mathrm{e}}=R_{\mathrm{e}}{ }^{2} / R_{\mathrm{d}}{ }^{2} \gtrsim 80 \gg 1$. As discussed in the Introduction, it is expected that the bubble dynamics then follows diffusion-limited growth.

Experimental Observations: Electrolysis-Induced Bubbles. Figure 3 shows the typical evolution of the bubble radius for a succession of bubbles captured during the first hour of constant $j$. Several effects come into view. First, the bubble growth rates eventually increase with time. This stands to reason because imposing a constant flux $J_{\mathrm{w}}$ leads to an accumulation of dissolved $\mathrm{H}_{2}$ gas in the bubble surroundings. In other words, there is a net increase of the average $\mathrm{H}_{2}$ concentration in the region where the bubble grows. The fact that repetitive, steady-state growth is not reached immediately is very striking and significant. The time required to reach the steady state in the absence of bubbles (Appendix A) can be very roughly estimated as $t_{\mathrm{ss}} \approx H^{2} / D_{\mathrm{H}_{2}}$, where $D_{\mathrm{H}_{2}}=4.2 \times 10^{-9} \mathrm{~m}^{2} / \mathrm{s}$ is the diffusivity of $\mathrm{H}_{2}$ in the electrolyte. ${ }^{39}$ To do so, we have assumed a 1D concentration profile of $C(z, t)$ with a constant flux at $z=0$ together with a zero-concentration boundary condition at the free surface, $C(H$, $t)=0$. Taking $H=10 \mathrm{~mm}$, this gives approximately $6 \mathrm{~h}$. In our experiments, it will be seen that the steady state is attained somewhat faster, within the order of $0.5-3 \mathrm{~h}$.

Second, the radius dynamics are affected by transient effects. This is especially evident in the very first bubble(s) of Figure $3 a-d$, whose growth dynamics displays fast temporal variations. This is attributed to the unsteadiness and nonuniformity of the evolving concentration field, which makes it more susceptible to perturbations during the first minutes after the onset of electrolysis. In time, these transient effects diminish as the boundary layer of evolved $\mathrm{H}_{2}$ gas grows and the local concentration field surrounding the pillar becomes more uniform. The effect of the contact angle dynamics on bubble growth is discarded because the detachment radius is always found to be below the maximum theoretical Fritz radius ${ }^{40}$ calculated using the pit circumference as the reference contact line. This implies that bubbles do not adhere to the top surface of the pillar. Instead, they are attached to the inside of the pit and detach through a necking process. ${ }^{33}$

Third, electrolytically driven bubble evolution is not perfectly reproducible for a number of reasons that will be discussed later. Premature detachment of particular bubbles is a commonly observed phenomenon in our experiments, especially for large current densities or supersaturations, as seen in Figure $3 \mathrm{~d}$ for $t=24$ and $35 \mathrm{~min}$.

Experimental Observations: Pressure-Controlled Bubbles. It is of interest to see how the growth dynamics obtained in the electrolysis experiments compares with those found in pressurecontrolled (P-C) experiments entailing a succession of $\mathrm{CO}_{2}$ bubbles produced in uniformly supersaturated water. To do so, a silicon chip, 
(a)

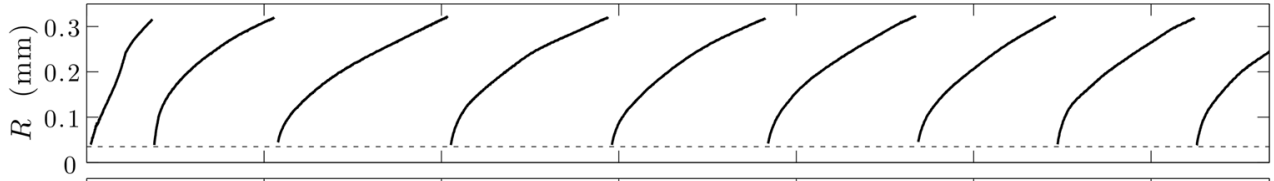

(b)

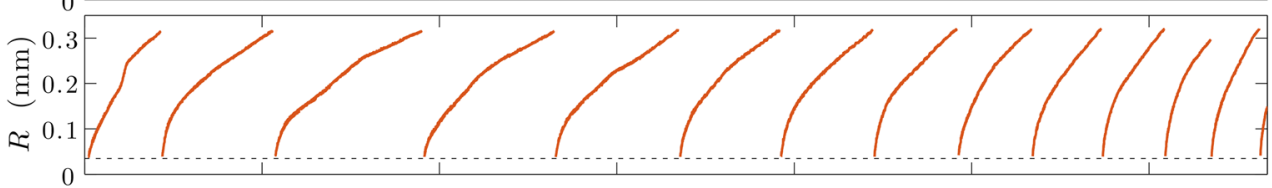

(c)

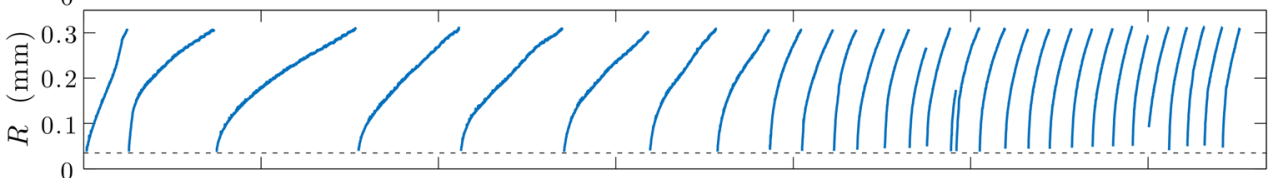

(d)

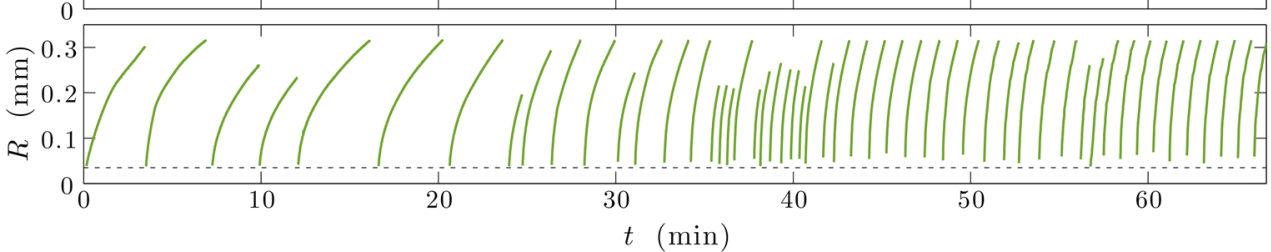

Figure 3. Time evolution of the bubble radius for a succession of bubbles captured during the first hour after the start of the electrolysis. Each individual curve represents a distinct bubble. Four different experiments are shown, in which the supplied current density is set to (a) 5.2, (b) 7.8, (c) 10.4 , and (d) $13.0 \mathrm{~A} / \mathrm{m}^{2}$. The pit and pillar radii are 5 and $15 \mu \mathrm{m}$, respectively, whereas the pillar height is $30 \mu \mathrm{m}$. Using $R_{\mathrm{d}} \approx 0.3 \mathrm{~mm}$ and $R_{\mathrm{e}}=3.5$ mm yields a Damköhler number of $D_{\mathrm{e}}=R_{\mathrm{e}}^{2} / R_{\mathrm{d}}^{2} \approx 136$. The dotted horizontal line marks the etching defect size below which it was not possible to optically detect the bubbles.

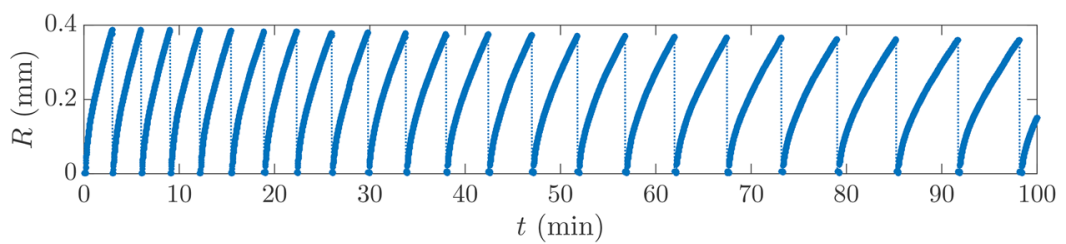

Figure 4. Pressure-controlled growth for a succession of $\mathrm{CO}_{2}$ bubbles for $\zeta=0.17$ during the first 100 min after the pressure drop. The dotted vertical lines indicate bubble detachment and nucleation of the following bubble. The pit and pillar radii are 10 and $15 \mu \mathrm{m}$, respectively, whereas the pillar height is $30 \mu \mathrm{m}$.

identical to those used in electrolysis experiments, is placed in the center of a pressurized test chamber (pressure $P_{\mathrm{s}} \approx 9$ bar) that is then filled with carbonated water previously saturated under the same pressure. A detailed description of the experimental setup and procedure may be found in the works by Enríquez et al. ${ }^{41}$ and Moreno Soto et al., ${ }^{33}$ to which the reader is referred. At the beginning of the experiment, the pressure is lowered to a value $P_{0} \approx 8 \mathrm{bar}$, which yields a supersaturation of $\zeta=P_{s} / P_{0}-1 \approx 0.17$. Shortly afterward, a single bubble nucleates and grows on top of the micropillar. As in the case of the electrolysis experiments, a succession of bubbles then follows. The time evolution of the bubble radii for a particular experiment is shown in Figure 4.

Two differences become immediately apparent upon comparison of Figures 3 and 4. In the first place, the P-C growth rate and detachment radius are more repeatable and stable than for the electrolysis experiments. In the second place, in the P-C experiments, the time taken for each bubble to detach grows with time, and thus the growth coefficient decreases. This is in contrast to what is observed in electrolysis, where the continuous dissolution of $\mathrm{H}_{2}$ in the bubble surroundings enhances the growth rate. In the case of P-C bubble growth, Moreno Soto et al. ${ }^{33}$ showed that the slowing down of the bubble growth is caused by the local depletion of $\mathrm{CO}_{2}$ near the bubble. Such a depletion phenomenon was attributed to buoyancy-driven convection and mixing due to bubble detachment.

Numerical Model. As a means to provide further insight into the growth dynamics of the electrolytic bubbles studied here, a numerical model for the growth of a spherical bubble attached to a micropillar of height $H_{\mathrm{p}}$ protruding from a (gas-evolving) substrate has been developed. It is an extension of the (pillarless) numerical model described in Peñas-López et al..$^{42}$ The governing equations are solved through a second-order finite-difference discretization in space and an implicit Euler method in time. The 2D axisymmetric grid is portrayed in Figure 5. The grid is divided into two regions that use different coordinate systems: a bulk region for $z>H_{\mathrm{p}}$ and a microlayer region below. In the bulk region, the advection-diffusion equation coupled with the streamfunction-vorticity equations ${ }^{42}$ are solved, imposing a no-slip boundary condition at $z=H_{\mathrm{p}}$. In the microlayer region, only the diffusion equation is solved. The concentration and its gradient are matched at the coupling plane $z=H_{\mathrm{p}}$. If required, a constant and uniform molar flux can be imposed at the substrate wall $(z=0)$ and pillar surface. Details about the simulation equations may be found in the Supporting Information.

The limitations of the simulation regarding the electrolysis experiments are that it considers neither the real finite size of the electrode nor the presence of a cavity (Figure 2). Moreover, we assume a spatially uniform flux along the electrode. Finally, we must estimate the initial $\mathrm{H}_{2}$ field corresponding to a bubble nucleating at some time after the start of electrolysis. To do so, we use the $1 \mathrm{D}$ analytical concentration profile in the absence of bubbles provided in Appendix A. Thus, this idealized initial concentration profile depends on just two input parameters, namely, the effective current density (which establishes the flux of dissolved $\mathrm{H}_{2}$ gas that is evolved at the electrode) and the elapsed time of electrolysis. We must finally remark that when modeling the growth of electrolytic bubbles a binary system of $\mathrm{H}_{2}$ gas and air is considered. The electrolyte is not degassed; in fact, it was kept in a container openly exposed to ambient air. Hence, we make the approximation that the electrolyte surrounding the micropillar is perfectly equilibrated with air at the beginning of bubble 


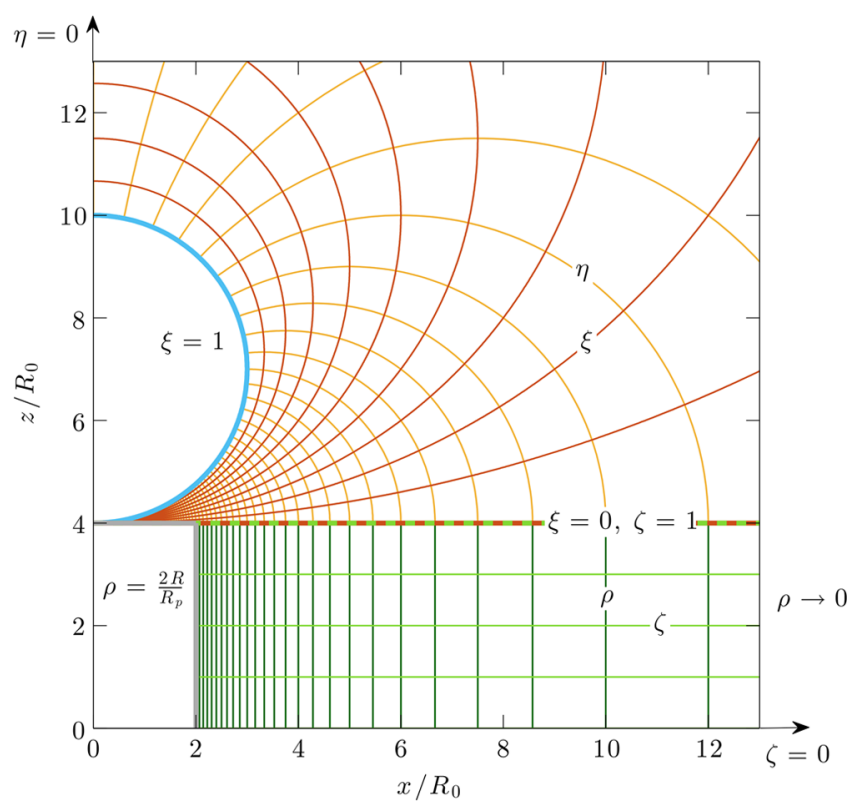

Figure 5. Schematic of the simulation grid for a pillar with dimensions $H_{\mathrm{p}} / R_{0}=4$ and $R_{\mathrm{p}} / R_{0}=2$. The simulation points fall on the intersection of the contour lines. The simulation is axisymmetric around the $z$ axis. The bulk region in tangent-sphere coordinates $(\eta, \xi)$ applies to $z>H_{\mathrm{p}}$. The microlayer region in pseudocylindrical coordinates $(\rho, \zeta)$ applies to $0<z<H_{\mathrm{p}}$. A matching condition is imposed at $z=H_{\mathrm{p}}$. Coordinates $\eta, \xi$, and $\rho$ evolve with the bubble so that $\xi=1$ (in blue) always maps the bubble surface, regardless of the actual bubble size. Here, the bubble size has been arbitrarily set to $R$ / $R_{0}=3$. The separation of the contours is uniform. Here, $\Delta \eta=\Delta \xi=$ $\Delta \rho=0.1$ and $\zeta=0.5$. Coordinate $\eta=0$ lies on the $z$ axis, $\eta \rightarrow \infty$, at the contact point.

growth. The presence of dissolved air explains why the first hydrogen bubbles nucleate and grow just a few seconds after the start of electrolysis when the electrolyte close to the electrode is still undersaturated in $\mathrm{H}_{2}$. Nonetheless, the small molar fractions of air typically present in the bubbles $(<15 \%)$ can be neglected for all other purposes.

\section{RESULTS AND DISCUSSION}

We begin this section with a theoretical description of the key quantity used to characterize the bubble growth dynamics, namely, the diffusive growth coefficient. The experimental results are then discussed, giving special attention to the effect that the different sources of depletion have on the measured growth coefficients. For the electrolysis experiments, the time evolution of the growth coefficients allows us to distinguish two sequential stages: an initial stagnation regime, where the bubble growth rates are nearly constant or slower than that of the very first bubbles, and a later supersaturation regime, where the bulk liquid contains enough $\mathrm{H}_{2}$ to sustain large bubble growth rates. Finally, the growth dynamics of each stage is quantitatively described.

Significance of the Diffusive Growth Coefficient. Assuming diffusion-limited growth, the $n$th bubble in the succession grows as $R_{n}\left(t_{n}\right)=b_{n} \sqrt{t_{n}}$, where $b_{n}$ is its associated diffusive growth coefficient and $t_{n}=t-t_{0, n}$ is the current time after nucleation at $t=t_{0, n}$. In the spirit of the theories for monocomponent bubble growth by Epstein and Plesset ${ }^{28}$ or Scriven, ${ }^{29}$ it is convenient to recast this law in terms of the dimensionless growth coefficient $\tilde{b}_{n}=b_{n} / \sqrt{ } D$ such that

$$
R_{n}\left(t_{n}\right)=\tilde{b}_{n} \sqrt{D t_{n}}
$$

where $D$ denotes the gas diffusivity. Indeed, if the bubble grows to a radius $R_{n}$ much larger than the size of the nucleation site (the pit on the pillar in our case), then its growth will be given by the Epstein-Plesset equation ${ }^{28}$ that determines the quasistatic diffusive growth of an isolated spherical bubble:

$$
\frac{\mathrm{d} R_{n}}{\mathrm{~d} t_{n}}=\frac{D R_{\mathrm{u}} T_{\infty}}{P_{0}}\left(\bar{C}_{n}-k_{\mathrm{H}} P_{0}\right)\left[\frac{1}{R_{n}}+\frac{1}{\sqrt{\pi D t_{n}}}\right]
$$

In this expression, $R_{\mathrm{u}}$ and $k_{\mathrm{H}}$ are the universal gas constant and Henry's constant, respectively. Moreover, $T_{\infty}$ and $P_{0}$ are the liquid temperature and pressure during growth, and $\bar{C}_{n}$ is the gas concentration far away from the bubble. Finally, note that surface tension has been neglected, as even the largest capillary (or Laplace) pressure $2 \gamma_{\mathrm{lg}} / R \approx 0.05$ bar is negligible in comparison to the pressure involved in bubble growth, $P_{0} \geq 1$ bar. Here, we have considered surface tension $\gamma_{\mathrm{lg}}=0.07 \mathrm{~N} / \mathrm{m}$ and the smallest trackable bubble size, $R=30 \mu \mathrm{m}$.

In the case of electrolysis within our air-equilibrated electrolyte, the Epstein-Plesset equation will be valid for sufficiently large concentrations of $\mathrm{H}_{2}$ such that the flux of other species $\left(\mathrm{N}_{2}, \mathrm{O}_{2}\right)$ into the growing bubble is small in comparison. In such cases, the ratio of $\mathrm{H}_{2}$-to-air fluxes can be shown to be roughly equivalent to $D_{\mathrm{H}_{2}} k_{\mathrm{H}, \mathrm{H}_{2}} \zeta /\left(D_{\text {air }} k_{\mathrm{H}, \text { air }}\right) \approx 2 \zeta$, where $\zeta$ is the supersaturation of $\mathrm{H}_{2}$, to be defined in eqs $7 \mathrm{a}$ and $7 \mathrm{~b}$.

At times $t_{n} \gg R_{0}^{2} / D$, this corresponds to roughly $t_{n} \gtrsim 1 \mathrm{~s}$ in our experiments. Equation 4 allows for the following solution (neglecting surface tension):

$$
R_{n}\left(t_{n}\right) \approx \sqrt{J a_{n} / \pi}\left[\sqrt{J a_{n}}+\left(2 \pi+J a_{n}\right)^{1 / 2}\right] \sqrt{D t_{n}}
$$

This solution is essentially that provided by Epstein and Plesset $^{28}$ formulated for the $n$th bubble in the succession and, correspondingly, using the mass-transfer Jakob number ${ }^{14,39}$ related to the $n$th bubble,

$$
J a_{n}=\frac{R_{\mathrm{u}} T_{\infty}}{P_{0}}\left(\bar{C}_{n}-k_{\mathrm{H}} P_{0}\right)=\Lambda \zeta_{n}
$$

The Jakob number is a measure of the driving force for bubble growth induced by a solubility parameter $\Lambda$, commonly known as the Ostwald coefficient, and the degree of supersaturation $\zeta_{n}$. These are defined as follows:

$$
\begin{aligned}
& \Lambda=k_{\mathrm{H}} R_{\mathrm{u}} T_{\infty} \\
& \zeta_{n}=\frac{\bar{C}_{n}}{k_{\mathrm{H}} P_{0}}-1
\end{aligned}
$$

We expect the Jakob number to be smaller than unity in our experiments. Indeed, for the P-C experiments, multiplying the supersaturation of $\mathrm{CO}_{2}\left(\zeta_{n} \approx 0.17\right)$ by the corresponding solubility tabulated in Table 1 results in $J a_{n} \approx 0.14$. For the $\mathrm{H}_{2}$ electrolysis experiments, $\zeta_{n}<12$ according to simulations; therefore, $J a_{n}<0.23$. Equation 5 then simplifies to

$$
R_{n}\left(t_{n}\right)=\sqrt{2 J a_{n} D t_{n}}
$$

because $J a_{n} \ll 2 \pi$ always holds. Within this diffusive regime, the Jakob number can be readily shown to be equivalent to the Péclet number, $P e_{n}=R_{n} \dot{R}_{n} / D$, which expresses the relative significance of advective over diffusive mass transport. The quantity $\dot{R}_{n}=\mathrm{d} R_{n} / \mathrm{d} t_{n}$ can be computed by differentiating eq 8 
Table 1. Gas Properties under Our Experimental Conditions $\left(T_{\infty}=20^{\circ} \mathrm{C}\right)$

\begin{tabular}{llll} 
& \multicolumn{1}{c}{$\mathrm{CO}_{2}$} & \multicolumn{1}{c}{$\mathrm{H}_{2}$} & \multicolumn{1}{c}{ air $^{c}$} \\
$D\left(\mathrm{~m}^{2} / \mathrm{s}\right)$ & $1.78 \times 10^{-9 a}$ & $4.2 \times 10^{-9 b}$ & $2.0 \times 10^{-9 b}$ \\
$k_{\mathrm{H}}\left(\mathrm{mol} \mathrm{m}^{-3} \mathrm{~Pa}^{-1}\right)$ & $3.36 \times 10^{-4}$ & $7.7 \times 10^{-6}$ & $8.5 \times 10^{-6}$ \\
$\Lambda$ & 0.824 & 0.0188 & 0.0207
\end{tabular}

${ }^{a}$ Pressure $\approx 8$ bar. ${ }^{b}$ Pressure $=1$ bar. ${ }^{c}$ Air is considered only in the simulation.

with respect to time. One then gets that $P e_{n} \equiv J a_{n}$, which is consistent with the fact that the Péclet number must also be smaller than unity. Finally, the direct comparison of eq 8 with eq 3 gives that the square of the dimensionless growth coefficient scales as the Jakob number:

$$
\tilde{b}_{n}^{2}=2 J a_{n}=2 \Lambda \zeta_{n}
$$

It should be pointed out that, strictly speaking, eq 9 is valid only for a spherical bubble far from any boundary. Nonetheless, taking into account the presence of the substrate will not modify any of the scalings but will change only the numerical coefficient relating $\tilde{b}_{n}^{2}$ and $J a_{n}$, as shown by Enríquez et al. ${ }^{27}$ for $\mathrm{P}-\mathrm{C}$ growth. In P-C growth, this coefficient is expected to be lowered by the presence of the inert substrate, which has a hindering effect on bubble growth. ${ }^{27,42}$ This is perceived upon inspection of the $\mathrm{CO}_{2}$ concentration field displayed in Figure $6 \mathrm{~d}$ obtained from simulation. The concentration isocontours are farther apart closer to the substrate compared to those near the apex.

The opposite occurs for the first bubbles in the case of electrolysis-driven growth, where $\mathrm{H}_{2}$ transfer rates (concentration gradients) are highest in the portion of the bubble interface located closest to the electrode, as can be inferred from the accumulation of isoconcentration lines in the region closer to the substrate (Figure 6a,b). Here, the bubbles are set to nucleate shortly after the onset of electrolysis; consequently, the $\mathrm{H}_{2}$ supersaturation in the bulk electrolyte is still low. One may note that as the degree of supersaturation in the bulk liquid increases with time (Figure $6 \mathrm{a}$ to $6 \mathrm{~b}$ to $6 \mathrm{c}$ ), the boundary layer evolves to the form attained in the P-C case (Figure 6d). Most of the $\mathrm{H}_{2}$ flux occurs at the apex of the bubble, as observed for $\mathrm{P}-\mathrm{C}$ growth.

Finally, we must remark that the pillar height $H_{\mathrm{p}}$ is much smaller than the radius of the growing bubble except for the initial seconds of bubble growth. Therefore, the effect of the pillar height on the aforementioned coefficient is expected to be small.

Growth Dynamics. The experimental diffusive growth coefficient for each electrolytic bubble in the succession was obtained by fitting the experimental data to eq 3, namely, $R_{n}\left(t_{n}\right)=\tilde{b}_{n} \sqrt{D t_{n}}$. More specifically,

$$
\tilde{\beta}_{\mathrm{H}_{2}}^{2}=\frac{1}{D_{\mathrm{H}_{2}}}\left\langle\frac{\mathrm{d} R^{2}}{\mathrm{~d} t}\right\rangle_{R \rightarrow R_{\mathrm{d}}}
$$

Here, $\tilde{\beta}_{\mathrm{H}_{2}}$ is the asymptotic growth coefficient ${ }^{13}$ at which the bubble reaches its detachment size. Even so, $\tilde{\beta}_{\mathrm{H}_{2}}$ should be regarded, by all accounts, as the experimentally obtained $\tilde{b}$ for the electrolytic bubbles.

The theoretical fits are plotted in Figure 7 for three different time periods after the start of electrolysis. Three regimes with distinct growth dynamics can be identified depending on the elapsed time of electrolysis. At early times, we devise a characteristic stagnation regime (Figure 7a), in which the second and third bubbles usually grow slower than the very first. Here, the accumulation of $\mathrm{H}_{2}$ gas that one would expect from the continuous flux at the electrode is in fact exhausted or overridden by local gas depletion effects induced by the growth of the first bubbles and other sources that will be later discussed. The duration of this stagnation regime is shortened with increasing current density (Figure 9). It stands to reason that no depletion regime may be observed if the current density is sufficiently large.

Thus, provided that the current density is large enough, the flow of $\mathrm{H}_{2}$ into the bubble liquid surroundings eventually overrides the flow of gas lost through depletion effects. This
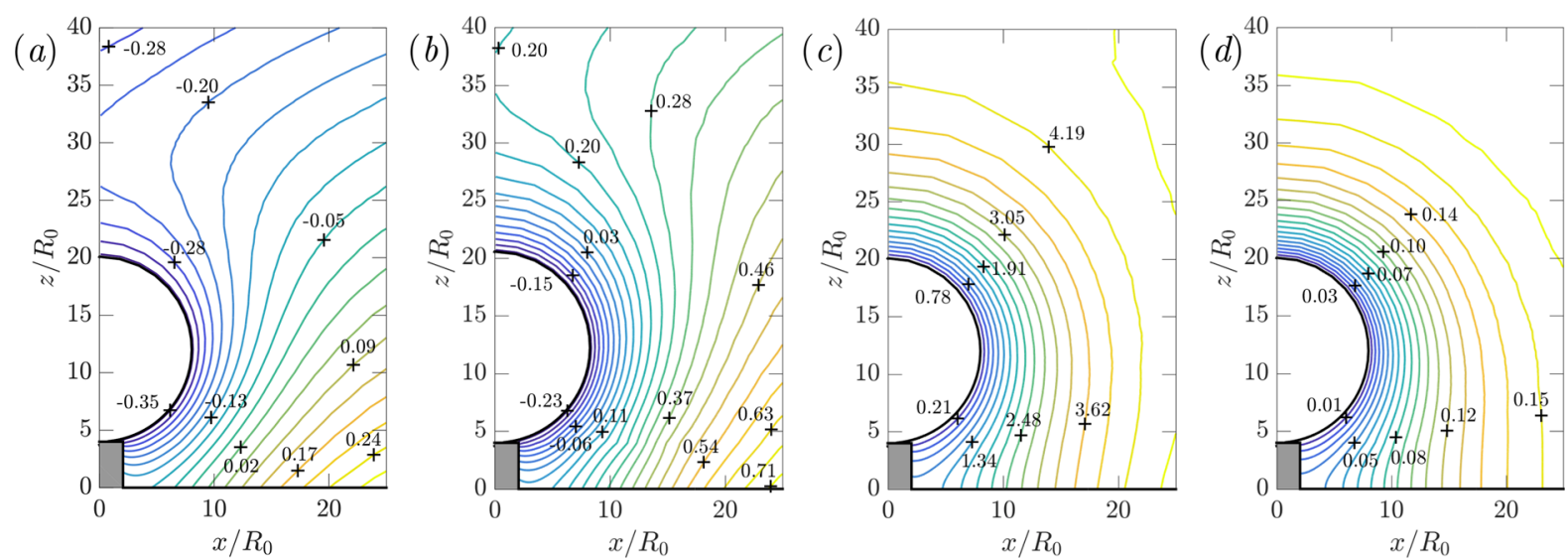

Figure 6. Simulation snapshots of $(\mathrm{a}-\mathrm{c}) \mathrm{H}_{2}$ or $(\mathrm{d}) \mathrm{CO}_{2}$ concentration isocontours around four distinct growing bubbles. The contour labels display the local supersaturation value, $C /\left(k_{\mathrm{H}} P_{0}\right)-1$. The simulations are performed for $R_{0}=7.5 \mu \mathrm{m}, R_{\mathrm{p}}=15 \mu \mathrm{m}$, and $H_{\mathrm{p}}=30 \mu \mathrm{m}$. The snapshots correspond to the early growth stage of the bubbles when $R=2 \mathrm{H}_{\mathrm{p}}=60 \mu \mathrm{m}$. Panels a-c show $\mathrm{H}_{2}$ bubbles growing in an air-saturated electrolyte assuming an effective current density of $1.56 \mathrm{~A} / \mathrm{m}^{2}$ that provides a similar $\mathrm{H}_{2}$ flux as the highest nominal current density used in our experiments (Figure 14.) The bubble nucleation time after the start of electrolysis, $t_{0}$, the corresponding initial degree of $\mathrm{H}_{2}$ supersaturation (in the absence of bubbles), $\zeta$, and the time after nucleation at which the snapshot is taken, $t$, are (a) $t_{0}=30 \mathrm{~s}, \zeta \approx 0, t \approx 31 \mathrm{~s}$, (b) $t_{0}=2 \mathrm{~min}, \zeta \approx 1, t \approx 16 \mathrm{~s}$, and (c) $t_{0}$ $=20 \mathrm{~min}, \zeta \approx 5.3, t \approx 3 \mathrm{~s}$. Panel $(\mathrm{d})$ portrays a $\mathrm{CO}_{2}$ bubble growing in an initially uniform supersaturated solution, $\zeta=0.17$ at $P_{0}=7.75$ bar. The bubble is $t \approx 5 \mathrm{~s}$ old. 
(a)

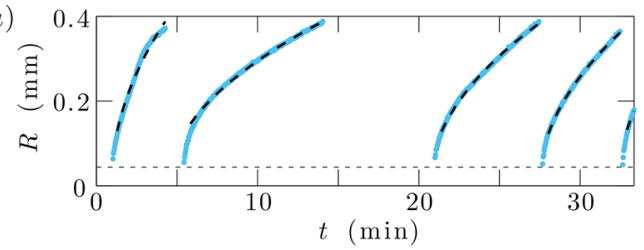

(c)

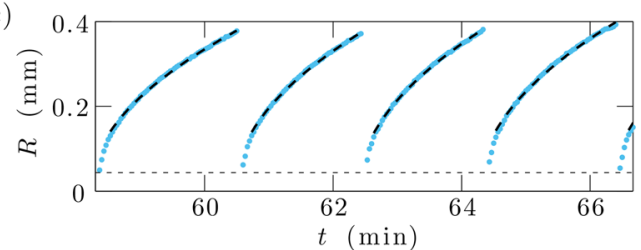

(e)

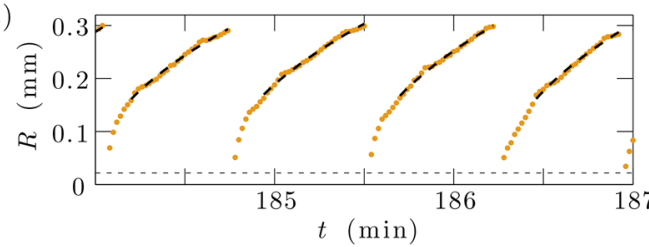

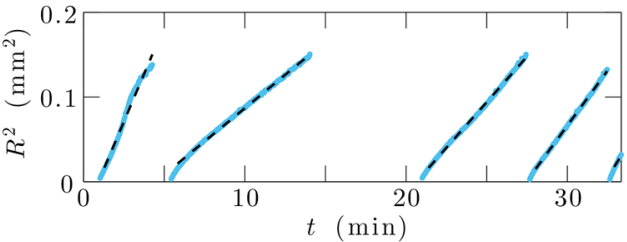

(d)
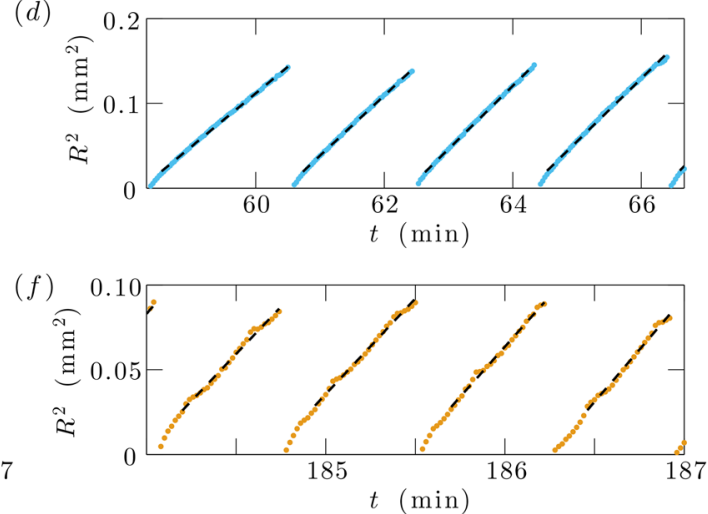

Figure 7. Bubble radius dynamics at three different time periods after the onset of electrolysis with $7.8 \mathrm{~A} / \mathrm{m}^{2}$. The measured bubble radii (dots) are plotted in ( $a, c$, and e), whereas the corresponding square of the measured radii are plotted in (b, d, and f). Panels (a and b) correspond to the stagnation regime, ( $c$ and $d$ ) correspond to the supersaturation regime, and (e and f) correspond to the advected growth regime. The dashed black curves are the fits from which the asymptotic growth coefficient $\tilde{\beta}_{\mathrm{H}_{2}}$ is obtained. The pit and pillar radius are 5 and $15 \mu \mathrm{m}$, respectively. The plots in (a-d) belong to the same experiment where $H_{\mathrm{p}}=30 \mu \mathrm{m}$. The plots in (e and $\mathrm{f}$ ) belong to a different experiment where $H_{\mathrm{p}}=15 \mu \mathrm{m}$. The dotted horizontal line marks the size below which it was not possible to optically determine the size of the bubble accurately.

(a)

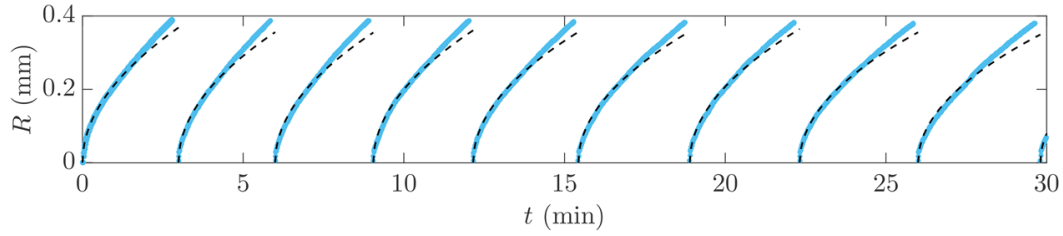

(b)

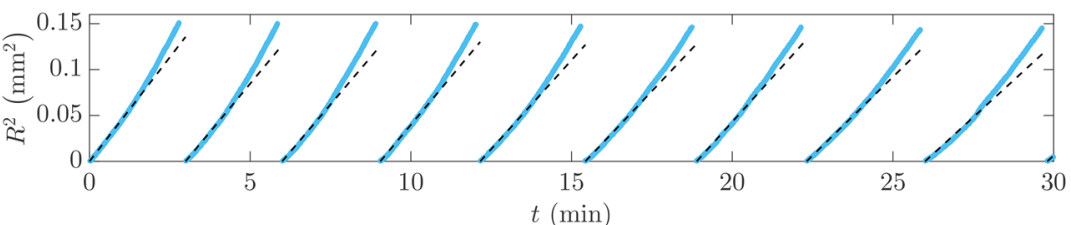

Figure 8. (a) Pressure-controlled bubble radius dynamics and (b) the corresponding squared radius. The dashed curves are the (extrapolated) fits from which $\tilde{\beta}_{\mathrm{CO}_{2}}$ is computed. The pit and pillar radii are 10 and $15 \mu \mathrm{m}$, respectively, whereas the pillar height is $30 \mu \mathrm{m}$.

results in a net accumulation of $\mathrm{H}_{2}$ and, consequently, a vertical $\mathrm{H}_{2}$ concentration boundary layer that develops from the electrode.

In such a case, a supersaturation regime (Figure $7 c$ ) is eventually attained. The local supersaturation of the surrounding electrolyte is now more uniform. The expected diffusive behavior, $R_{n}^{2} \propto t_{n}$, can be corroborated in Figure $7 \mathrm{~d}$. If, at later times, the supersaturation becomes large enough, an advective growth regime (Figure 7e) is manifested. It is characterized by discontinuous $\mathrm{d} R / \mathrm{d} t$ dynamics caused by the appearance of boundary-induced advection in bubble growth and departure. ${ }^{32}$ Furthermore, a fast initial growth is observed. It is attributed to the presence of a thin boundary layer of excess $\mathrm{H}_{2}$ gas near the gas-evolving electrode that quickly gets adsorbed into the bubble. As a consequence, the initial growth is observed to be more pronounced for pillar heights of 0 or $15 \mu \mathrm{m}$ as opposed to $30 \mu \mathrm{m}$.
In slightly supersaturated water- $-\mathrm{CO}_{2}$ solutions, bubbles are known to diffusively grow before density-driven convection sets in during the final stages of their growth. ${ }^{27}$ As a consequence, the diffusive growth coefficient for the $\mathrm{CO}_{2}$ bubbles in the P-C experiments was similarly computed as

$$
\tilde{\beta}_{\mathrm{CO}_{2}}^{2}=\frac{1}{D_{\mathrm{CO}_{2}}}\left\langle\frac{\mathrm{d} R^{2}}{\mathrm{~d} t}\right\rangle_{R<3 R_{\mathrm{d}} / 4}
$$

where $R_{\mathrm{d}}$ denotes the bubble radius at detachment. The upper limit of $3 R_{\mathrm{d}} / 4$ is established in order to restrict the fitting of $\tilde{\beta}_{\mathrm{CO}_{2}}$ to just the initial diffusive growth regime.

The theoretical fit (extrapolated to span the full bubble lifetime $)$ is compared to a typical experiment $(\zeta=0.17)$ in Figure 8. The initial diffusive growth, where $R \propto \sqrt{ } t$, can be perfectly distinguished. One may also note a deviation between the theoretical fits and experiments as the bubble radius reaches its detachment size. This deviation is expected because it is 
related to the onset of natural convection at the end of bubble growth. $^{27,33}$ The prominent effect of density-induced convection on bubble growth observed here is, on the other hand, negligible for the case of $\mathrm{H}_{2}$ bubbles.

The computation of $\tilde{\beta}_{\mathrm{H}_{2}}$ and $\tilde{\beta}_{\mathrm{CO}_{2}}$ for each individual bubble essentially allows for a quantitative measure of the evolution of the growth rate of the bubble succession as a whole. The fact that the evolution of $\tilde{\beta}_{\mathrm{H}_{2}}$ in time for a succession of bubbles under constant-current electrolysis is far from uniform is immediately corroborated in Figure 9. An illustrative movie of

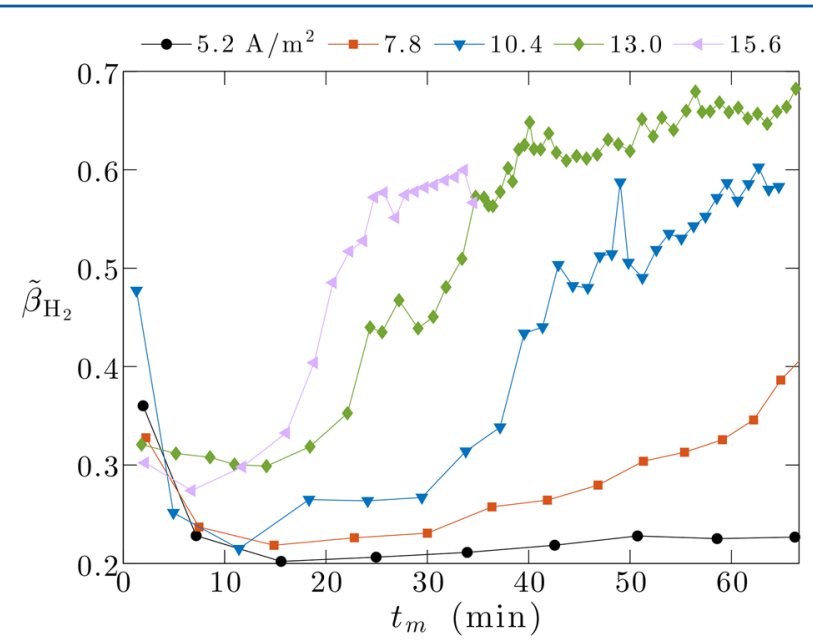

Figure 9. $\tilde{\beta}_{\mathrm{H}_{2}}$ for a succession of electrolytic bubbles exposed to constant current densities. Each point corresponds to a distinct bubble in the succession. Time $t_{\mathrm{m}}$ is the mean time (after the start of electrolysis) of the fitting period used to compute $\tilde{\beta}_{\mathrm{H}_{2}}$. The bubbles evolved on a pit with a radius of $5 \mu \mathrm{m}$, a pillar radius of $15 \mu \mathrm{m}$, and a pillar height of $30 \mu \mathrm{m}$.

the electrolysis experiment with $j=10.4 \mathrm{~A} / \mathrm{m}^{2}$ can be found in the Supporting Information. In the video, the time evolution of the bubble radii and the growth coefficient are also plotted in real time.

The first few bubbles fall under what we have referred to as the stagnation regime, in which $\tilde{\beta}_{\mathrm{H}_{2}}$ is observed to decrease or remain fairly constant. The stagnation regime occurs as a result of the partial depletion of the evolving boundary layer of dissolved gas. There are three main sources of depletion that will soon be described. These are responsible for the everpresent loss of gas dissolved in the cavity where the electrode is held (cf. Figure 2). This loss is most noticeable and even dominant in the early stages of electrolysis. This stands to reason because, as mentioned before, the local concentration of dissolved $\mathrm{H}_{2}$ gas near the pillar is relatively small at the beginning of the electrolysis. Accordingly, gas losses due to depletion constitute a proportionally large reduction of the small amount of gas dissolved.

The subsequent bubbles experience a fast increase in $\tilde{\beta}_{\mathrm{H}_{2}}$. This is a sign of the net accumulation of dissolved $\mathrm{H}_{2}$ gas in the cavity. In other words, the flow rate of evolved $\mathrm{H}_{2}$ into the cavity overrides the smaller flow rate losses due to depletion. Figure 10 displays the same experimental values of $\tilde{\beta}_{\mathrm{H}_{2}}$ as a function of the total electric charge that has been in circulation before a given time, which is a direct measure of the total mass of $\mathrm{H}_{2}$ supplied. For a given charge $Q=j t$, during the initial

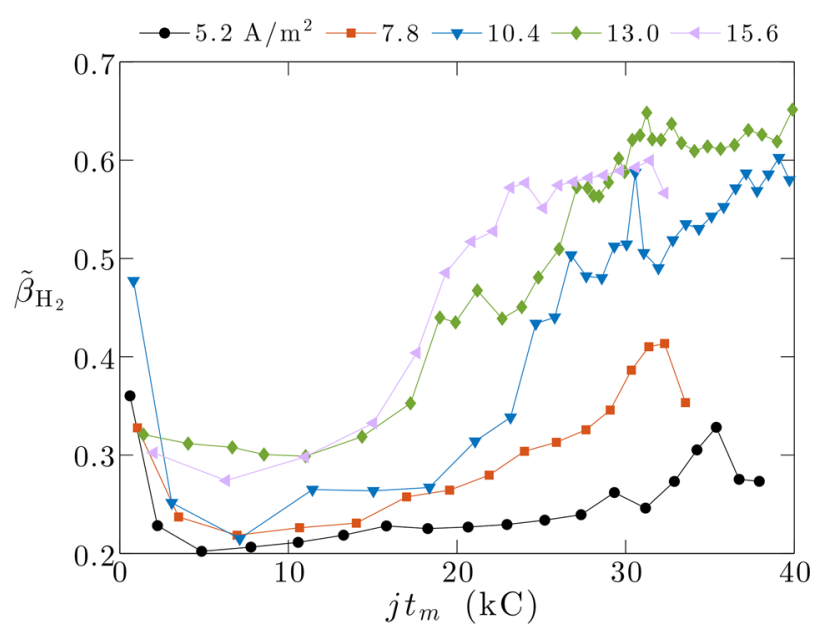

Figure 10. Diffusive growth coefficient as a function of charge for different current densities (caption of Figure 9). The efficiency of gas evolution in the transient regime increases with the current density $j$.

transient stage of electrolysis, we conclude that $\tilde{\beta}_{\mathrm{H}_{2}}$ is higher for larger current densities. It is expected that the gas evolution efficiency increases with current density because the diffusion length of dissolved $\mathrm{H}_{2}$, which scales as $\sqrt{D_{\mathrm{H}_{2}} Q / j}$, is smaller. In other words, mass is supplied faster and $\mathrm{H}_{2}$ does not diffuse fast enough into the bulk electrolyte above the cavity in which the bubble evolves. Consequently, the gas supersaturation in the cavity surrounding the electrode is higher.

Naturally, in the P-C experiments, the absence of gas production must translate into a permanent depletion regime. Moreno Soto et $\mathrm{al}^{33}$ recently developed a theoretical depletion model for this particular scenario. On the basis of this model (Appendix B), the evolution of the experimental growth coefficient should approximately satisfy

$$
\frac{1}{\tilde{\beta}_{\mathrm{CO}_{2}}^{2}(t)}-\frac{1}{\tilde{\beta}_{\mathrm{CO}_{2}}^{2}(0)}=\frac{2 \pi}{3} \frac{D_{\mathrm{CO}_{2}} t}{R_{\mathrm{d}}{ }^{2}}
$$

The experimental evolution in time of $\tilde{\beta}_{\mathrm{CO}_{2}}$ is plotted in Figure 11(a). In Figure 11(b), these are compared to the best fitting law $1 / \tilde{\beta}_{\mathrm{CO}_{2}}^{2}(t)=m t+c$. According to eq 12 , the slope is $m=$ $0.027 \mathrm{~s}^{-1}$. This estimate agrees well with experiments, where $m$ $\approx 0.03 \mathrm{~s}^{-1}$. Similarly, constant $c=2.93$ from theory falls close to the experimental values that lie within 2.6-2.9. The small differences may be associated with the many simplifications and approximations that the theoretical model is based upon. Nonetheless, the good overall agreement between the model and experiments indeed suggests that in the P-C configuration there is only depletion from previous bubbles in the succession.

Depletion Sources in the Electrolysis Experiments. In our electrolysis experiments, however, the electrolyte in the cavity suffers $\mathrm{H}_{2}$ gas depletion from (i) previous bubbles in the succession, from (ii) parasitic bubbles (eventually millimetersized) forming all around the circular sidewall of the cavity (Figure 12), and from (iii) the cavity opening, toward which $\mathrm{H}_{2}$ is transported by diffusion. Regarding the latter, it is worth noticing that the cavity perimeter is much smaller than the perimeter of the holder above (cf. Figure 2). In addition, the volume of electrolyte in the tank above the cavity is almost 3 orders of magnitude larger than the volume of the cavity itself. The opening of the cavity therefore initially acts as a zero $\mathrm{H}_{2}$ 

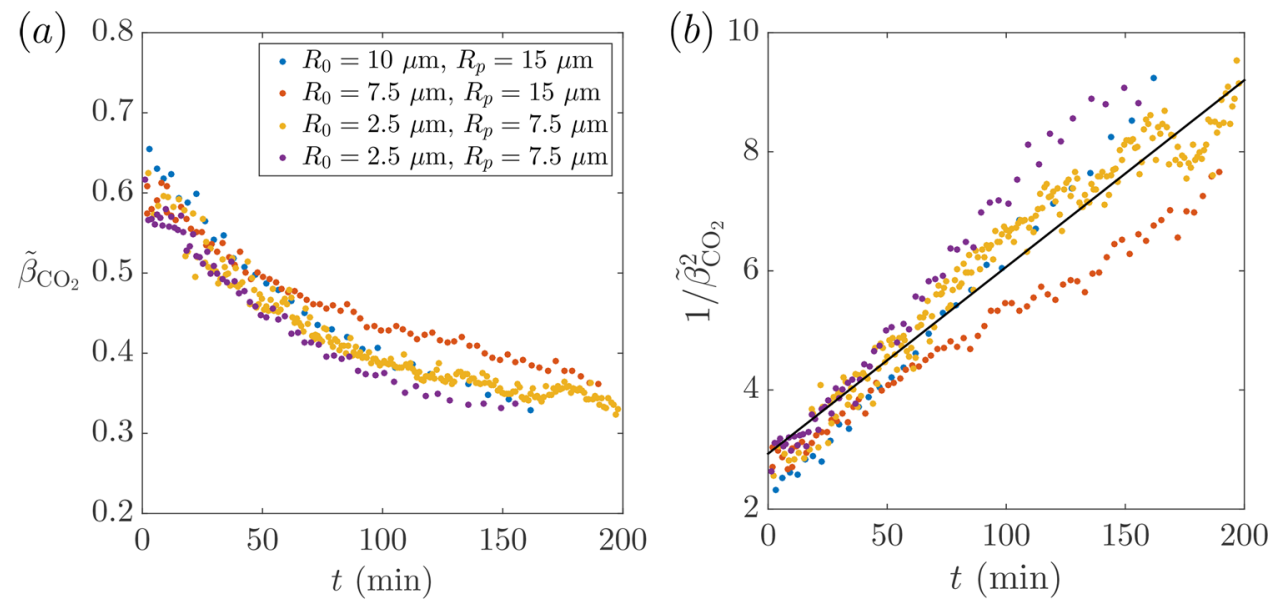

Figure 11. (a) Diffusive growth coefficient, $\tilde{\beta}_{\mathrm{CO}_{2}}$, as a function of time for four different P-C experiments. The time measurement for each bubble $n$ is associated with its moment of detachment. (b) Plot of $1 / \tilde{\beta}_{\mathrm{CO}_{2}}^{2}$ as a function time. The straight solid line shows linear eq 12 , with a slope of $m=$ $2 \pi D_{\mathrm{CO}_{2}} t /\left(3 R_{\mathrm{d}}^{2}\right)=0.027 \mathrm{~s}^{-1}$.

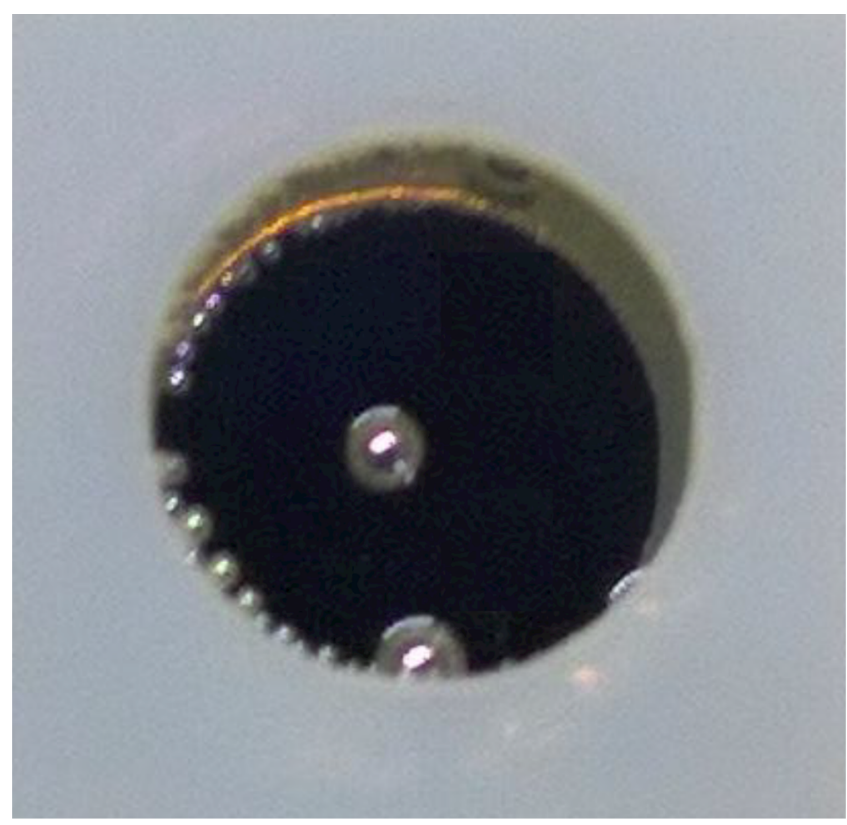

Figure 12. Photograph showing the parasitic bubbles forming on the circular corner where the electrode (black surface) meets the sidewall of the cavity and at the sharp upper edge of the cavity wall (cf. the large bottommost bubble). These bubbles visibly surround the bubble that forms on top of the micropillar in the center of the electrode. The electrode diameter is $7 \mathrm{~mm}$.

concentration boundary condition (a pseudofree surface) because $\mathrm{H}_{2}$ is essentially diffusing into an infinite reservoir.

The effect of these depletion sources on the bubble growth dynamics will be discussed next for the electrolysis experiments, discerning between the stagnation regime and supersaturation (eventually steady-state) regime that follows.

Stagnation Regime. In the stagnation regime, the bulk concentration of dissolved $\mathrm{H}_{2}$ gas within the cavity is small. We can then make the rough approximation that the $\mathrm{H}_{2}$ gas in these bubbles is entirely supplied by the molar flux $J_{\mathrm{w}}$ that is evolved within a harvest area $\pi \delta^{2}$ on the flat electrode. The characteristic harvest length $\delta$ is the effective electrode length required to sustain a bubble growing at a particular rate in a degassed liquid and assuming a gas evolution efficiency of unity.
Consequently, $\delta$ is always smaller than the electrode radius, $\delta \leq$ $R_{\mathrm{e}}$ (or an equivalent length). Indeed, the aforementioned depletion sources tend to decrease the growth rate and hence also $\delta$. Thus, $\delta$ constitutes an important design parameter in electrolytic systems and should be investigated in future studies. In fact, $\delta / R_{\mathrm{e}}$ can be regarded as a direct measure of the gas evolution efficiency of the system.

Assuming then that these bubbles are entirely composed of $\mathrm{H}_{2}$, a simple mass balance scaling yields

$$
\frac{4 \pi}{3} \frac{P_{0}}{R_{\mathrm{u}} T_{\infty}} \frac{R_{\mathrm{d}}{ }^{3}}{\Delta t} \approx J_{\mathrm{w}} \pi \delta^{2}
$$

where $\Delta t$ denotes the bubble lifetime prior to detachment. As a first approximation, we expect the harvest length to scale as $\delta \approx$ $R_{\mathrm{d}}$. If we further assume purely diffusive growth, $R_{\mathrm{d}}=\tilde{\beta}_{\mathrm{H}_{2}} \sqrt{D_{\mathrm{H}_{2}} \Delta t}$, and note that $J_{\mathrm{w}} \propto j$, then eq 13 suggests that the asymptotic growth rate must scale as $\tilde{\beta}_{\mathrm{H}_{2}}{ }^{2} \approx j R_{\mathrm{d}}$. This relation is compared against experimental values in Figure 13. The experimental dispersion is expected as a result of the random variability of the harvest length (primarily due to the spatial nonuniformity of the $\mathrm{H}_{2}$ flux at the electrode) and the depletion strength from one experiment to the other.

Within the stagnation regime or transient regime that follows, the diffusive depletion at the cavity opening has an

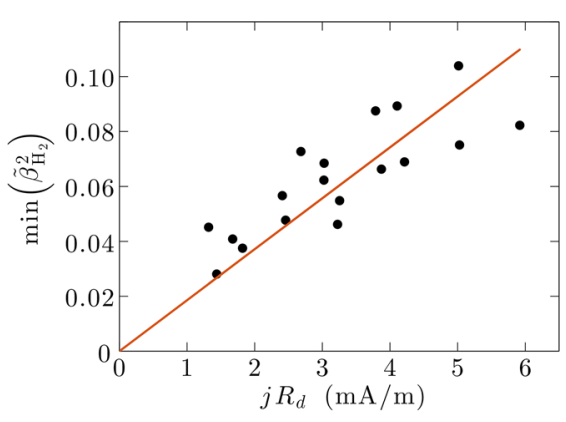

Figure 13. Minimum squared value of $\tilde{\beta}_{\mathrm{H}_{2}}$ plotted versus the product of current density and detachment radius. Each point corresponds to a single bubble in a unique succession. The linear regression fit is also plotted. 
- experiment - w/ cavity effect ---w/o cavity effect
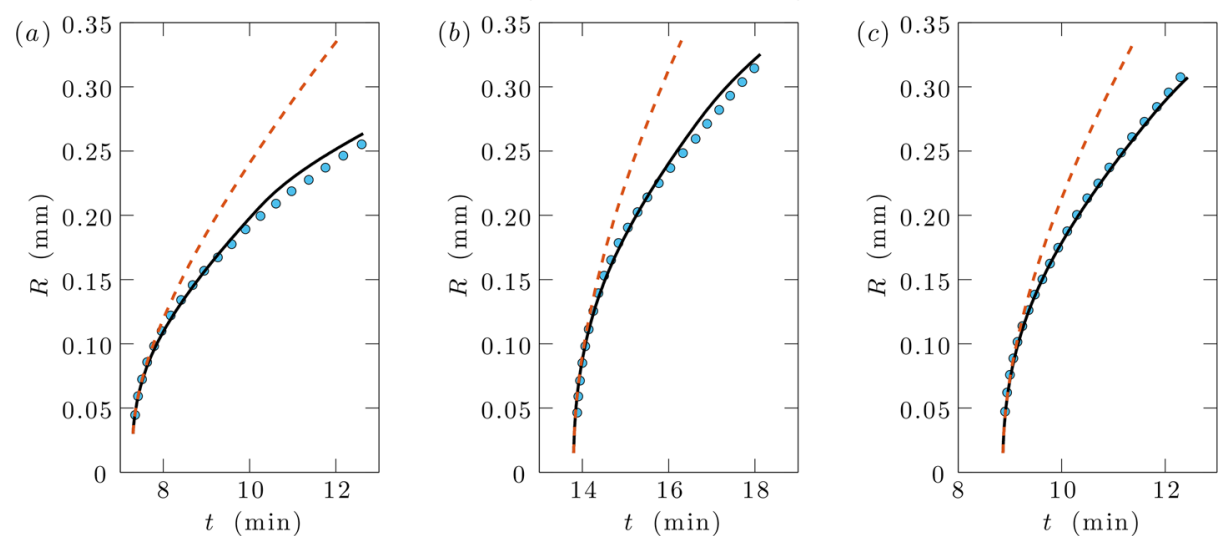

Figure 14. Comparison between simulation (curves) and the experimental growth dynamics of the third bubble for three nominal current densities: (a) 7.8, (b) 10.4, and (c) $15.6 \mathrm{~A} / \mathrm{m}^{2}$. Simulations employ effective current densities of (a) 1.04 , (b) 1.30 , and (c) $1.56 \mathrm{~A} / \mathrm{m}^{2}$. Using eq 17 in Appendix A, these yield initial supersaturations, $\zeta$, of approximately (a) 1.5, (b) 3.3, and (c) 3.1. The black curves are computed by imposing a zeroconcentration boundary condition at heights of (a) 1.0, (b) 1.3, and (c) $1.3 \mathrm{~mm}$ to model the effect of the cavity. The red dashed curves are computed without imposing the aforementioned boundary condition.

important retardation effect on the bubble growth rate as the bubble grows larger and gets closer to the opening. This retardation can be noted upon inspection of Figures 3 and 7 a. This claim is supported by numerical simulations as seen in Figure 14. The simulation curves predict the experimental behavior once a zero-concentration boundary condition is imposed at a height somewhat lower, but close to the actual cavity depth of $2 \mathrm{~mm}$. However, this is expected because a lower height also accounts for other losses such as parasitic bubble formation at the cylindrical sidewall of the cavity.

It is imperative to note that the effective current density (which establishes the molar flux of dissolved $\mathrm{H}_{2}$ at the electrode) employed in the simulation is in fact taken as a free parameter. The effective current density and the experimental time of nucleation of a particular bubble are put into eq 17 in appendix A to obtain the initial concentration field in which that particular bubble grows. The effective current density is thus chosen so that the numerical solution matches the experimental radii at the initial growth stage of the bubble. Surprisingly, the effective current densities are 7 to 10 times smaller than the nominal (experimental) values. Accordingly, the growth rates obtained by simulations at the nominal current densities were exceedingly high. A similar discrepancy in theoretical versus measured $\mathrm{H}_{2}$ diffusive fluxes was observed by Aoki et al., ${ }^{43}$ who argued that most of the gas may not actually dissolve in hydrated form. They reported that $\mathrm{H}_{2}$ gas preferentially resides in the form of stable bulk nanobubbles $(R \approx 400 \mathrm{~nm})$ instead. $^{43,44}$

Although at the moment we cannot give a precise answer to this discrepancy, we propose two plausible, not mutually exclusive, explanations. First, not all of the $\mathrm{H}_{2}$ flux put into dissolution remains in the electrolyte or eventually goes to the bubble nucleating at the pit. Indeed, we observe that large bubbles unavoidably form at the cylindrical cavity sidewall. Second, the numerical model assumes a spatially uniform current density along the substrate. In practice, this may not be so. In fact, the current density can be highly nonhomogeneous for planar electrodes. ${ }^{6}$ Moreover, one may note that the actual boundary condition occurring at the electrode is of constant potential and not of uniform current density. Thus, close to the base of the pillar the local current density must be smaller than the average on the electrode surface.
Supersaturation Regime. In the supersaturation regime, the gas concentration in the cavity is relatively more uniform at the beginning of the bubble growth, especially for sufficiently high supersaturations, $\bar{C}_{n} /\left(k_{\mathrm{H}} P_{0}\right) \gg 1$. By making use of eq $6, \bar{C}_{n}$ can be made continuous in time by setting $\bar{C}(t)$ equal to the wall concentration $C(z=0, t)$ in the absence of bubbles assuming a $1 \mathrm{D}$ profile. The expression is provided in eq 17 in Appendix A. In such a case, the growth coefficient exhibits the following behavior at long times:

$$
\tilde{\beta}_{\mathrm{H}_{2}}^{2}(t) \approx \frac{2 R_{\mathrm{u}} T_{\infty}}{P_{0}} \bar{C}\left(t ; H_{\text {eff }}, J_{\mathrm{w}, \mathrm{eff}}\right)
$$

The local concentration $\bar{C}(t)$ depends on the effective molar flux $J_{w, \text { eff }}$, or, equivalently, on the effective current density $j_{\text {eff }}$. These will be smaller than the nominal values in order to account for the aforementioned discrepancy between the experimental and theoretical concentrations. Furthermore, $\bar{C}(t)$ is also dependent on the effective electrolyte liquid column height $H_{\text {eff }}$ that is expected to be lower than $H=12$ $\mathrm{mm}$. $H_{\text {eff }}$ accounts for the geometry of the electrolysis holder and cavity and losses from growing and departing bubbles at the cavity perimeter. For high current densities, $H_{\text {eff }}$ is also limited by the maximum supersaturation of dissolved $\mathrm{H}_{2}$ that the electrolyte can withstand at the experimental pressure. The steady-state growth coefficient can be shown to directly depend on these two quantities:

$$
\tilde{\beta}_{\mathrm{H}_{2}, \mathrm{ss}}^{2} \approx \frac{2 R_{\mathrm{u}} T_{\infty} F v}{D_{\mathrm{H}_{2}} P_{0}} j_{\mathrm{eff}} H_{\mathrm{eff}}
$$

Figure 15 compares this theoretical model for the behavior of $\tilde{\beta}_{\mathrm{H}_{2}}$ at long times against three experiments with different pillar heights but performed under the same current density. A similar steady-state value is attained in all cases after about 150 min. However, it is evident that the transient behavior of $\tilde{\beta}_{\mathrm{H}_{2}}$ is not repetitive across experiments. The deviations arise presumably from differences in the flux distribution across the electrode samples and especially from gas losses from the random formation and subsequent detachment of large parasitic bubbles on the cavity sidewall. The bubbles in the succession must then compete against the parasitic bubbles for 


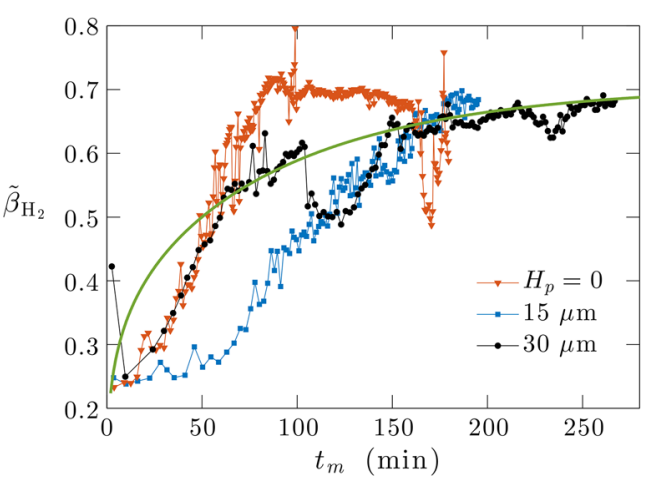

Figure 15. Evolution in time of the diffusion growth rate for $j=7.8 \mathrm{~A} /$ $\mathrm{m}^{2}$ for three different experiments. The pit radius is $R_{0}=5 \mu \mathrm{m}$ for $H_{\mathrm{p}}$ $=0$ and $30 \mu \mathrm{m}$, whereas $R_{0}=1 \mu \mathrm{m}$ for $H_{\mathrm{p}}=15 \mu \mathrm{m}$. The green curve is a theoretical approximate solution given in eq 14 , with $j_{\text {eff }}=1.04 \mathrm{~A} / \mathrm{m}^{2}$ (same value as in simulations) and $H_{\text {eff }}=8 \mathrm{~mm}$.

the available $\mathrm{H}_{2}$ gas. The formation of the parasitic bubbles ultimately has a hindering effect on the growth rates of the bubbles growing on the pillar. This effect increases over time as the solution becomes more supersaturated and the parasitic bubbles increase in size and hence in proximity with respect to the micropillar. For instance, the sudden decrease in $\tilde{\beta}_{\mathrm{H}_{2}}$ at around $110 \mathrm{~min}$ in the experiment with $H_{\mathrm{p}}=30 \mu \mathrm{m}$ in Figure 15 is a consequence of a particular large parasitic bubble that expanded quite close to the pillar. After its detachment at approximately $130 \mathrm{~min}, \tilde{\beta}_{\mathrm{H}_{2}}$ is observed to recover quickly.

The radius dynamics of two bubbles belonging to this particular succession are compared with simulations in Figure 16. The agreement is quite good once the (a priori unknown) initial supersaturation level $\zeta$ is adjusted. Note that $\zeta$ corresponds to the mean value of the initial concentration profile close to the electrode wall. As before, $\zeta$, or rather the initial concentration profile, is computed from eq 17 , and it is a
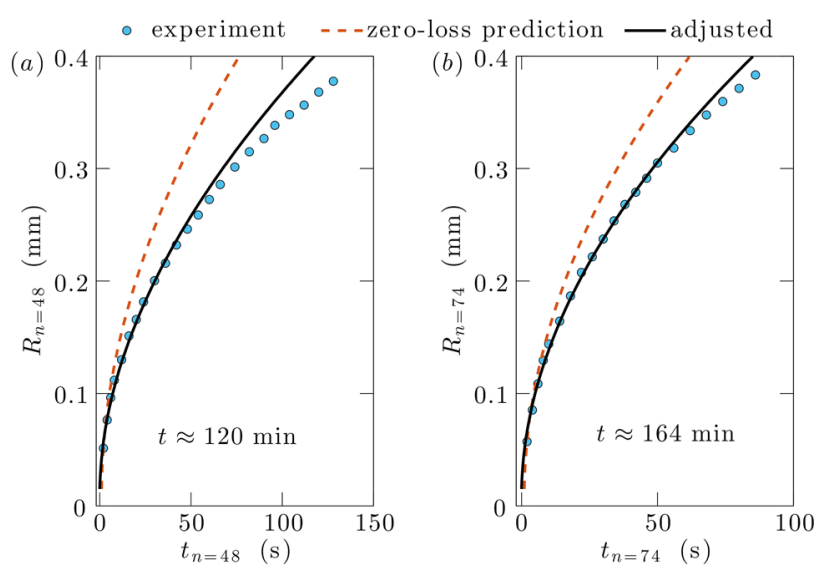

Figure 16. Comparison between simulation (curves) and the experimental growth dynamics for the (a) 48th and (b) 74th bubble of the succession with $H_{\mathrm{p}}=30 \mu \mathrm{m}$ (cf. Figure 15). The nominal current density is $j=7.8 \mathrm{~A} / \mathrm{m}^{2}$, whereas simulations employ an effective current density of $1.04 \mathrm{~A} / \mathrm{m}^{2}$. The red dashed curves are expected growth for a bubble in an infinitely long electrode that nucleates at the experimental time $t$ in the absence of any previous bubbles. The initial concentration profile is given by eq 17 evaluated at time $t$. The resulting local supersaturations are $\zeta_{48} \approx 9.25$ and $\zeta_{74} \approx$ 11.0. For the black curves, the time is adjusted to (a) $0.5 t$ and (b) $0.6 t$, resulting in $\zeta_{48} \approx 7.25$ and $\zeta_{74} \approx 8.4$. function of the current density and time of nucleation after the onset of electrolysis. The value of the effective current density employed is $1.04 \mathrm{~A} / \mathrm{m}^{2}$, the same as the one obtained by fitting the initial growth rate of the third bubble in the succession (as done for Figure 14a). Thus, the nucleation time after the onset of electrolysis is now the sole free parameter. Setting it equal to the experimental nucleation time clearly overestimates the growth rates, as seen from the zero-loss prediction curves in Figure 16. One can then account for depletion losses by decreasing the time of nucleation used to calculate $\zeta$. After a suitable reduction in the nucleation time and hence $\zeta$, the bubble growth behavior is reasonably well reproduced.

One may note that in the supersaturation regime the zeroconcentration boundary condition is not required because the entire cavity, and possibly a small region above it, will be homogeneously supersaturated to a good degree. As the bubble approaches departure, a retardation in its growth is noticeable nonetheless. This is attributed to $\mathrm{H}_{2}$ deprivation due to the presence of parasitic bubbles. The deviation for the 48th bubble is greater than for the 74th bubble. This is consistent with the fact that the 48th bubble is deprived from the $\mathrm{H}_{2}$ available to a greater extent by the aforementioned large parasitic bubble.

\section{CONCLUSIONS}

The growth dynamics of a succession of $\mathrm{H}_{2}$ bubbles driven by constant-current electrolysis on a large electrode has been studied. The bubbles form within a hydrophobic pit on top of a single micropillar etched on a flat silicon electrode. The large surface area of the electrode ensures that the bubble growth is diffusion-limited. As such, growth can be suitably described in terms of an experimentally determined dimensionless growth coefficient $\tilde{\beta}_{\mathrm{H}_{2}}$ according to $R(t)=\tilde{\beta}_{\mathrm{H}_{2}} \sqrt{D_{\mathrm{H}_{2}} t}$.

The accumulation of dissolved $\mathrm{H}_{2}$ near the gas-evolving electrode is hindered by several depletion sources. We identify depletion from (i) previous bubbles in the succession, from (ii) unwanted parasitic bubbles forming on the cavity sidewall, and from (iii) the mere presence of the cavity where the electrode is being held. It follows that the degree of supersaturation near the electrode (and consequently $\widetilde{\beta}_{\mathrm{H}_{2}}$ ) is largely unsteady. For the range of current densities considered in our experiments $\left(5-15 \mathrm{~A} / \mathrm{m}^{2}\right)$, the $\mathrm{H}_{2}$ bubble succession first experiences a stagnation regime where depletion effects are most noticeable. Here, $\tilde{\beta}_{\mathrm{H}_{2}}$ remains fairly constant or decreases. A fast growth in $\tilde{\beta}_{\mathrm{H}_{2}}$ then follows before the steady state is reached. Conversely, for the case of $\mathrm{CO}_{2}$ bubbles in our P-C experiments, persistent depletion (exclusively from previous bubbles in the succession) is observed. The analogous growth coefficient $\tilde{\beta}_{\mathrm{CO}_{2}}$ decreases in such a way that the quantity $1 / \tilde{\beta}_{\mathrm{CO}_{2}}^{2}(t)$ increases linearly with time.

Furthermore, in the electrolysis experiments, the depletion effect of the cavity opening and of parasitic bubbles forming on the cavity sidewall accounts for the growth retardation observed as bubbles approach their departure size. Oppositely, $\mathrm{CO}_{2}$ bubbles experience enhanced growth as they approach detachment. This is attributed to the onset of density-induced natural convection.

The comparison of both case scenarios serves to highlight the important effect of depletion, omnipresent in the growth of a succession of bubbles. The knowledge obtained can be further expanded by conducting electrolysis experiments with two main variations. First, we have nucleated bubbles within a cavity with 
no flow (stagnant conditions). In real-life scenarios, however, it may be desirable to have continuous flow. This will change the force balance keeping the bubbles pinned at the surface and influence mass transport through advection. Second, closed systems that could be pressurized (as opposed to open systems, such as the one presented here) will be very similar to the actual utilization of microfluidic electrochemical reactors. ${ }^{3}$ Both cases are expected to entail distinct bubble growth dynamics and certainly warrant further study.

\section{APPENDIX A}

\section{One-Dimensional Concentration Profile from a Gas-Evolving Surface}

The one-dimensional diffusion equation governing the evolution of the dissolved gas concentration profile

$$
\frac{\partial C}{\partial t}=D \frac{\partial^{2} C}{\partial z^{2}}
$$

applies to the liquid region $0<z<H$. A constant molar flux $J_{\mathrm{w}}$ enters the region at $z=0$. At the free surface, $C(z=H, t)=0$. Initially, we assume that $C(z, t=0)=0$. The concentration profile has the following analytical solution: ${ }^{45}$

$$
\begin{gathered}
C(z, t)=2 J_{\mathrm{w}} \sqrt{t / D} \sum_{n=0}^{\infty}(-1)^{n}\left\{\operatorname{ierfc}\left(\frac{n \mathrm{H}+z / 2}{\sqrt{D t}}\right)\right. \\
\left.-\operatorname{ierfc}\left(\frac{(n+1) H-z / 2}{\sqrt{D t}}\right)\right\}
\end{gathered}
$$

where the integral of the complementary error function is defined as

$$
\operatorname{ierfc}(x)=\int_{x}^{\infty} \operatorname{erfc}(u) \mathrm{d} u=\frac{\mathrm{e}^{-x^{2}}}{\sqrt{\pi}}-x \operatorname{erfc}(x)
$$

Equation 17 converges in time to the linear profile of the steady-state solution, $C(z, t \rightarrow \infty)=J_{\mathrm{w}}(H-z) / D$. Profiles computed from eq 17 are plotted in Figure 17, whereas the evolution in time of the concentration at the gas-evolving surface $(z=0)$ is plotted in Figure 18. Note that for the case of infinite height, $H \rightarrow \infty$, the solution in eq 17 simplifies to

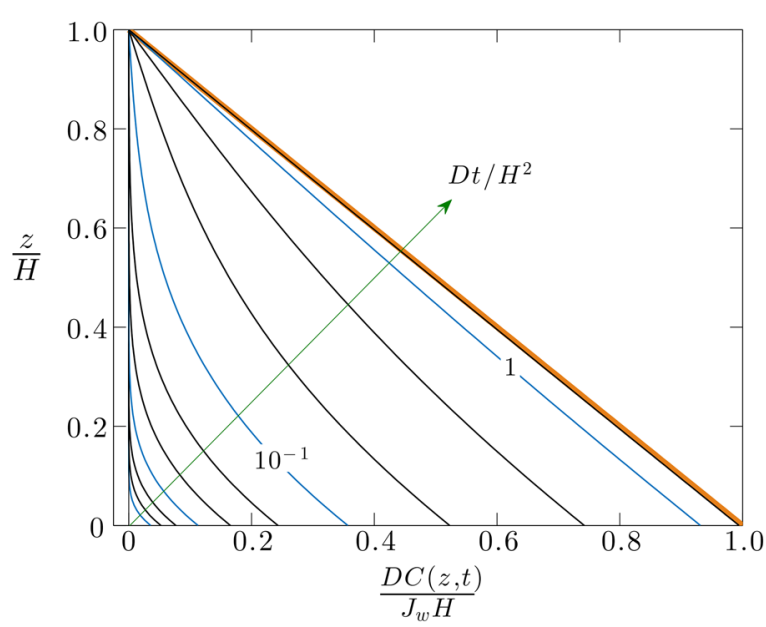

Figure 17. Universal plot of the time evolution of the 1D concentration profiles for different instants in dimensionless time. The profiles marked in blue correspond to $\log _{10}\left(D t / H^{2}\right)=-3,-2,-1$, and 0 . The profile in red is the steady-state profile.

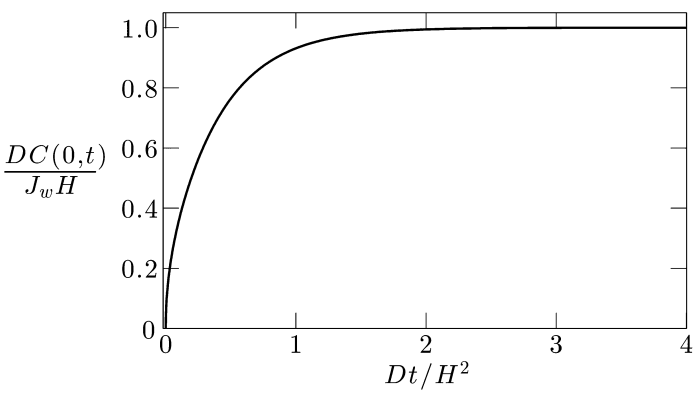

Figure 18. Invariant, dimensionless plot of the time evolution of the concentration at the electrode wall $(z=0)$.

$$
C(z, t)=2 J_{\mathrm{w}} \sqrt{t / D} \operatorname{ierfc}\left(\frac{z}{\sqrt{4 D t}}\right)
$$

In this particular case, no steady-state solution exists, and the concentration at the wall increases unboundedly as $2 J_{\mathrm{w}} \sqrt{t /(\pi D)}$. Furthermore, if small heights or short times are considered such that $z / \sqrt{4 D t} \ll 1$, then eq 19 can be approximated as

$$
C(z, t)=\frac{2 J_{\mathrm{w}}}{\sqrt{\pi D}}\left(\sqrt{t}-\frac{z}{2} \sqrt{\pi / D}\right)
$$

\section{APPENDIX B}

\section{Depletion Model for the Growth Coefficient}

Let subscript $n$ refer to the $n$th bubble. Let $t_{n}=t-t_{0, n}$, where $t_{0, n}$ is the nucleation time of such a bubble and $t_{0, n=1}=0$. Let $\theta_{n}(r, t)=C(r, t)-\bar{C}_{n}$, where

$$
\bar{C}_{n}=C_{n}\left(r, t_{n}=0\right)=C_{n-1}\left(r=\delta_{n-1}, t_{n-1}=\Delta t_{n-1}\right)
$$

is the characteristic (homogeneous) concentration field (left behind by bubble $n-1$ ) in which bubble $n$ immediately grows. It is essentially the concentration field evaluated at the characteristic diffusion length $\delta_{n}$ and time $\Delta t_{n}$ corresponding to the previous bubble, where $\Delta t_{n}=t_{\mathrm{d}, n}-t_{0, n}$ is the bubble residence time. Note that we assume a negligible time between detachment and nucleation of the subsequent bubble: $t_{n-1}=$ $\Delta t_{n-1}$ is equivalent to $t_{n}=0$. Furthermore, the detachment radius $R_{\mathrm{d}}=\tilde{b}_{n} \sqrt{D \Delta t_{n}}$ is assumed to be constant for all bubbles.

Following the model proposed by Moreno Soto et al., ${ }^{33}$ we treat each bubble in the succession as a point source of molar strength

$$
\dot{n}_{n}=-\frac{4 \pi P_{0} R_{\mathrm{d}}{ }^{3}}{3 R_{\mathrm{u}} T_{\infty} \Delta t_{n}}
$$

which acts as a boundary condition to the diffusion equation,

$$
\frac{\partial \theta_{n}}{\partial t_{n}}=\frac{D}{r^{2}} \frac{\partial}{\partial r}\left(r^{2} \frac{\partial \theta_{n}}{\partial r}\right)
$$

together with a second boundary condition $\theta_{n}(r \rightarrow \infty, t)=0$ and initial condition $\theta_{n}\left(r, t_{n}=0\right)=0$. Let $\bar{\theta}_{n}$ be the characteristic solution for $\theta_{n}$ evaluated at $r=\delta_{n}$ and $t=\Delta t_{n}$. Then,

$$
\bar{\theta}_{n}=-\frac{4 \pi P_{0} R_{\mathrm{d}}}{3 R_{\mathrm{u}} T_{\infty}} \frac{\tilde{b}_{n}^{2}}{\delta_{n}} \operatorname{erfc}\left(\frac{\delta_{n} \tilde{b}_{n}}{2 R_{\mathrm{d}}}\right)
$$


Let $\Delta \bar{C}_{n}=\bar{C}_{n}-k_{\mathrm{H}} P_{0}$ be the characteristic concentration field strength relative to the interfacial concentration. One then has that

$$
\Delta \bar{C}_{n}=\bar{C}_{1}-k_{\mathrm{H}} P_{0}+\sum_{m=2}^{n} \bar{\theta}_{m}
$$

Making use of eq 9, we know that $\Delta \bar{C}_{n}=P_{0} \tilde{b}_{n}^{2} /\left(2 R_{u} T_{\infty}\right)$. Substituting this expression along with eq 24 into eq 25 yields

$$
\tilde{b}_{n}^{2}=\tilde{b}_{1}^{2}-\frac{8 \pi}{3} \sum_{m=2}^{n} R_{\mathrm{d}} \frac{\tilde{b}_{m}^{2}}{\delta_{m}} \operatorname{erfc}\left(\frac{\delta_{m} \tilde{b}_{m}}{2 R_{\mathrm{d}}}\right)
$$

where $\tilde{b}_{1}^{2}=2 R_{\mathrm{u}} T_{\infty} \Delta \bar{C}_{1} / P_{0}$ is just the known growth rate of the first bubble (corresponding to the initial, undepleted supersaturation $\left.\Delta \bar{C}_{1}\right)$. From experimental evidence in Moreno Soto et al., ${ }^{33}$ we can make the approximation that $\delta_{n} \approx 4 R_{\mathrm{d}}$. Moreover, because $\tilde{b}_{m} \approx 0.1$ is small, the complementary error function can be approximated as unity. Equation 26 then simplifies to

$$
\tilde{b}_{n}^{2}=\tilde{b}_{1}^{2}-\frac{2 \pi}{3} \sum_{m=2}^{n} \tilde{b}_{m}^{2}
$$

The above expression can be made continuous in time through

$$
\tilde{b}^{2}(t)=\tilde{b}^{2}(0)-\frac{2 \pi}{3} \int_{0}^{t} \tilde{b}^{2}\left(t^{\prime}\right) \frac{\mathrm{d} n}{\mathrm{~d} t^{\prime}} \mathrm{d} t^{\prime}
$$

where $\mathrm{d} n / \mathrm{d} t=D \tilde{b}^{2}(t) / R_{\mathrm{d}}^{2}$ is simply the continuous form of the bubble nucleation frequency. Differentiating eq 28 in time yields

$$
2 \tilde{b} \frac{\mathrm{d} \tilde{b}}{\mathrm{~d} t}=-\frac{2 \pi D}{3 R_{\mathrm{d}}{ }^{2}} \tilde{b}^{4}
$$

Upon integration, we obtain

$$
\frac{1}{\tilde{b}^{2}(t)}-\frac{1}{\tilde{b}^{2}(0)}=\frac{2 \pi}{3} \frac{D t}{R_{\mathrm{d}}{ }^{2}}
$$

\section{ASSOCIATED CONTENT}

\section{S Supporting Information}

The Supporting Information is available free of charge on the ACS Publications website at DOI: 10.1021/acs.langmuir.7b02978.

Formulation of the mass transfer problem and governing equations implemented in the simulations (PDF) Movie of a particular electrolysis experiment (AVI)

\section{AUTHOR INFORMATION}

\section{Corresponding Author}

*E-mail: d.vandermeer@utwente.nl.

\section{ORCID $\odot$}

Álvaro Moreno Soto: 0000-0002-9810-7759

Pablo Peñas-López: 0000-0002-3503-3342

Detlef Lohse: 0000-0003-4138-2255

David Fernández Rivas: 0000-0003-4329-3248

Notes

The authors declare no competing financial interest.

\section{ACKNOWLEDGMENTS}

The authors thank S. Schlautmann for his fruitful contribution to this work. This work was supported by The Netherlands Centre for Multiscale Catalytic Energy Conversion (MCEC), an NWO Gravitation programme funded by the Ministry of Education, Culture and Science of the government of The Netherlands. We also acknowledge the support of the Spanish Ministry of Economy and Competitiveness through grants DPI2014-59292-C3-1-P, DPI2015-71901-REDT, and DPI2017-88201-C3-3-R, partially funded through European Funds.

\section{REFERENCES}

(1) Tomasoni, F.; van Parys, H.; Terryn, H.; Hubin, A.; Deconinck, J.; Buchlin, J.-M.; van Beeck, J. Identification of bubble evolution mechanisms during AC electrograining. Electrochem. Commun. 2010, $12,156-159$.

(2) Nierhaus, T.; Van Parys, H.; Dehaeck, S.; van Beeck, J.; Deconinck, H.; Deconinck, J.; Hubin, A. Simulation of the two-phase flow hydrodynamics in an IRDE reactor. J. Electrochem. Soc. 2009, 156, P139-P148.

(3) Modestino, M. A.; Fernández Rivas, D.; Hashemi, S. M. H.; Gardeniers, J. G. E.; Psaltis, D. The potential for microfluidics in electrochemical energy systems. Energy Environ. Sci. 2016, 9, 33813391.

(4) Zeng, K.; Zhang, D. Recent progress in alkaline water electrolysis for hydrogen production and applications. Prog. Energy Combust. Sci. 2010, 36, 307-326.

(5) Zhang, L.; Zhang, Y.; Zhang, X.; Li, Z.; Shen, G.; Ye, M.; Fan, C.; Fang, H.; Hu, J. Electrochemically controlled formation and growth of hydrogen nanobubbles. Langmuir 2006, 22, 8109-8113.

(6) Svetovoy, V. B.; Sanders, R. G. P.; Elwenspoek, M. C. Transient nanobubbles in short-time electrolysis. J. Phys.: Condens. Matter 2013, $25,184002$.

(7) Chen, Q.; Luo, L.; White, H. S. Electrochemical generation of a hydrogen bubble at a recessed platinum nanopore electrode. Langmuir 2015, 31, 4573-4581.

(8) Nowotny, J.; Sorrell, C. C.; Sheppard, L. R.; Bak, T. Solarhydrogen: Environmentally safe fuel for the future. Int. J. Hydrogen Energy 2005, 30, 521-544.

(9) Walter, M. G.; Warren, E. L.; McKone, J. R.; Boettcher, S. W.; Mi, Q.; Santori, E. A.; Lewis, N. S. Solar water splitting cells. Chem. Rev. 2010, 110, 6446-6473.

(10) Gabrielli, C.; Huet, F.; Nogueira, R. Fluctuations of concentration overpotential generated at gas-evolving electrodes. Electrochim. Acta 2005, 50, 3726-3736.

(11) van der Linde, P.; Moreno Soto, Á.; Peñas-López, P.; RodríguezRodríguez, J.; van der Meer, D.; Lohse, D.; Huskens, J.; Gardeniers, H.; Fernández Rivas, D. Evolution of Electrolytic Hydrogen Bubbles on Microstructured Electrodes; 232nd ECS Meeting; National Harbor, MD, Oct 1-5, 2017.

(12) Kadyk, T.; Bruce, D.; Eikerling, M. How to enhance gas removal from porous electrodes? Sci. Rep. 2016, 6, 38780.

(13) Glas, J. P.; Westwater, J. W. Measurements of the growth of electrolytic bubbles. Int. J. Heat Mass Transfer 1964, 7, 1427-1443.

(14) Verhaart, H. F. A.; de Jonge, R. M.; van Stralen, S. J. D. Growth rate of a gas bubble during electrolysis in supersaturated liquid. Int. J. Heat Mass Transfer 1980, 23, 293-299.

(15) Brandon, N. P.; Kelsall, G. H. Growth kinetics of bubbles electrogenerated at microelectrodes. J. Appl. Electrochem. 1985, 15, 475-484.

(16) Brussieux, C.; Viers, P.; Roustan, H.; Rakib, M. Controlled electrochemical gas bubble release from electrodes entirely and partially covered with hydrophobic materials. Electrochim. Acta 2011, $56,7194-7201$ 
(17) Fernández, D.; Maurer, P.; Martine, M.; Coey, J. M. D.; Möbius, M. E. Bubble formation at a gas-evolving microelectrode. Langmuir 2014, 30, 13065-13074.

(18) Sakuma, G.; Fukunaka, Y.; Matsushima, H. Nucleation and growth of electrolytic gas bubbles under microgravity. Int. J. Hydrogen Energy 2014, 39, 7638-7645.

(19) Chandran, P.; Bakshi, S.; Chatterjee, D. Study on the characteristics of hydrogen bubble formation and its transport during electrolysis of water. Chem. Eng. Sci. 2015, 138, 99-109.

(20) Yang, X.; Karnbach, F.; Uhlemann, M.; Odenbach, S.; Eckert, K. Dynamics of single hydrogen bubbles at a platinum microelectrode. Langmuir 2015, 31, 8184-8193.

(21) Wang, Y.; Hu, X.; Cao, Z.; Guo, L. Investigations on bubble growth mechanism during photoelectrochemical and electrochemical conversions. Colloids Surf., A 2016, 505, 86-92. Sixth International Workshop on Bubble and Drop Interfaces.

(22) Leenheer, A. J.; Atwater, H. A. Water-splitting photoelectrolysis reaction rate via microscopic imaging of evolved oxygen bubbles. $J$. Electrochem. Soc. 2010, 157, B1290-B1294.

(23) Wang, Y.; Zaytsev, M. E.; The, H. L.; Eijkel, J. C. T.; Zandvliet, H. J. W.; Zhang, X.; Lohse, D. Vapor and gas-bubble growth dynamics around laser-irradiated, water-immersed plasmonic nanoparticles. ACS Nano 2017, 11, 2045-2051.

(24) Vogt, H. The rate of gas evolution of electrodes-I. An estimate of the efficiency of gas evolution from the supersaturation of electrolyte adjacent to a gas-evolving electrode. Electrochim. Acta 1984, 29, 167-173.

(25) Darby, R.; Haque, M. S. The dynamics of electrolytic hydrogen bubble evolution. Chem. Eng. Sci. 1973, 28, 1129-1138.

(26) Barker, G. S.; Jefferson, B.; Judd, S. J. The control of bubble size in carbonated beverages. Chem. Eng. Sci. 2002, 57, 565-573.

(27) Enríquez, O. R.; Sun, C.; Lohse, D.; Prosperetti, A.; van der Meer, D. The quasi-static growth of $\mathrm{CO}_{2}$ bubbles. J. Fluid Mech. 2014, 741, R1.

(28) Epstein, P. S.; Plesset, M. S. On the stability of gas bubbles in liquid-gas solutions. J. Chem. Phys. 1950, 18, 1505-1509.

(29) Scriven, L. E. On the dynamics of phase growth. Chem. Eng. Sci. 1959, 10, 1-13.

(30) Lohse, D.; Zhang, X. Surface nanobubbles and nanodroplets. Rev. Mod. Phys. 2015, 87, 981-1035.

(31) Janssen, L. J. J.; Hoogland, J. G. The effect of electrolytically evolved gas bubbles on the thickness of the diffusion layer. Electrochim. Acta 1970, 15, 1013-1023.

(32) Vogt, H.; Stephan, K. Local microprocesses at gas-evolving electrodes and their influence on mass transfer. Electrochim. Acta 2015, $155,348-356$.

(33) Moreno Soto, Á.; Prosperetti, A.; Lohse, D.; van der Meer, D. Gas depletion through single gas bubble diffusive growth and its effect on subsequent bubbles. J. Fluid Mech. 2017, 831, 474-490.

(34) Liu, X.; Coxon, P. R.; Peters, M.; Hoex, B.; Cole, J. M.; Fray, D. J. Black silicon: fabrication methods, properties and solar energy applications. Energy Environ. Sci. 2014, 7, 3223-3263.

(35) Du, C.; Gao, C.; Yin, G.; Chen, M.; Wang, L. Facile fabrication of a nanoporous silicon electrode with superior stability for lithium ion batteries. Energy Environ. Sci. 2011, 4, 1037-1042.

(36) Elbersen, R.; Tiggelaar, R. M.; Milbrat, A.; Mul, G.; Gardeniers, H.; Huskens, J. Controlled doping methods for radial $\mathrm{p} / \mathrm{n}$ junctions in silicon. Adv. Energy Mater. 2015, 5, 1401745.

(37) Bremond, N.; Arora, M.; Ohl, C.-D.; Lohse, D. Controlled multibubble surface cavitation. Phys. Rev. Lett. 2006, 96, 224501.

(38) Borkent, B. M.; Gekle, S.; Prosperetti, A.; Lohse, D. Nucleation threshold and deactivation mechanisms of nanoscopic cavitation nuclei. Phys. Fluids 2009, 21, 102003.

(39) Vogt, H. On the supersaturation of gas in the concentration boundary layer of gas evolving electrodes. Electrochim. Acta 1980, 25, $527-531$.

(40) Fritz, W. Berechnung des Maximal Volume von Dampfblasen. Phys. Z. 1935, 36, 379-388.
(41) Enríquez, O. R.; Hummelink, C.; Bruggert, G.-W.; Lohse, D.; Prosperetti, A.; van der Meer, D.; Sun, C. Growing bubbles in a slightly supersaturated liquid solution. Rev. Sci. Instrum. 2013, 84, 065111.

(42) Peñas-López, P.; Moreno Soto, Á.; Parrales, M. A.; van der Meer, D.; Lohse, D.; Rodríguez-Rodríguez, J. The history effect in bubble growth and dissolution. Part 2. Experiments and simulations of a spherical bubble attached to a horizontal flat plate. J. Fluid Mech. 2017, 820, 479-510.

(43) Aoki, K.; Toda, H.; Yamamoto, J.; Chen, J.; Nishiumi, T. Is hydrogen gas in water present as bubbles or hydrated form? J. Electroanal. Chem. 2012, 668, 83-89.

(44) Kikuchi, K.; Nagata, S.; Tanaka, Y.; Saihara, Y.; Ogumi, Z. Characteristics of hydrogen nanobubbles in solutions obtained with water electrolysis. J. Electroanal. Chem. 2007, 600, 303-310.

(45) Carslaw, H. S.; Jaeger, J. C. Conduction of Heat in Solids; Oxford University Press, 1959. 